NIST Technical Note 1995

\title{
Juliet 1.3 Test Suite: Changes From 1.2
}

Paul E. Black

This publication is available free of charge from: https://doi.org/10.6028/NIST.TN.1995

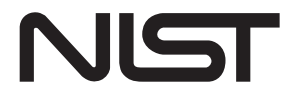

National Institute of Standards and Technology

U.S. Department of Commerce 


\title{
NIST Technical Note 1995
}

\section{Juliet 1.3 Test Suite: Changes From 1.2}

\author{
Paul E. Black \\ Software Quality Group \\ Software and Systems Division \\ Information Technology Laboratory
}

This publication is available free of charge from:

https://doi.org/10.6028/NIST.TN.1995

June 2018

INCLUDES UPDATES AS OF 08-22-2019; SEE PAGE iii

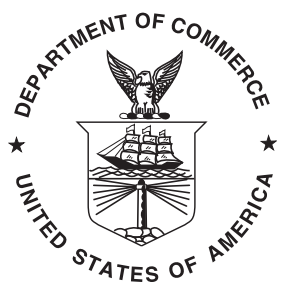

U.S. Department of Commerce Wilbur L. Ross, Jr., Secretary

National Institute of Standards and Technology Walter Copan, NIST Director and Undersecretary of Commerce for Standards and Technology 
Certain commercial entities, equipment, or materials may be identified in this document in order to describe an experimental procedure or concept adequately. Such identification is not intended to imply recommendation or endorsement by the National Institute of Standards and Technology, nor is it intended to imply that the entities, materials, or equipment are necessarily the best available for the purpose.

National Institute of Standards and Technology Technical Note 1995

Natl. Inst. Stand. Technol. Tech. Note 1995, 37 pages (June 2018)

CODEN: NTNOEF

This publication is available free of charge from: https://doi.org/10.6028/NIST.TN.1995 


\begin{abstract}
The Juliet test suite is a systematic set of thousands of small test programs in $\mathrm{C} / \mathrm{C}++$ and Java, exhibiting over 100 classes of errors, such as buffer overflow, OS injection, hardcoded password, absolute path traversal, NULL pointer dereference, uncaught exception, deadlock, and missing release of resource. These test programs should be helpful in determining capabilities of software assurance tools, particularly static analyzers, in Unix, Microsoft Windows, and other environments. Juliet was developed by the National Security Agency's Center for Assured Software and first released in December 2010. It has been enhanced twice since then. Version 1.2 was released in May 2013 with a total of 86864 test cases.

In the years after its release, many problems and deficiencies in Version 1.2 came to our attention. Released in October 2017, Version 1.3 fixes about fourteen systematic problems in Version 1.2 and adds tests for prefix and postfix increment integer overflow and decrement integer underflow. This technical note details the changes from Version 1.2 to 1.3. This note also lists known problems remaining in Juliet 1.3.
\end{abstract}

\title{
Key words
}

Buffer overflow; Bugs Framework (BF); Common Weakness Enumeration (CWE); cybersecurity; integer overflow; Juliet test suite; OS injection bugs; programming language test material; software assurance; software quality; static analysis; static source code analyzers. 


\section{Table of Contents}

1 Introduction 1

1.1 The Organization of the Juliet Suite of Test Cases 2

1.2 Summary of Changes and Remaining Issues 4

2 Details of All Changes 5

2.1 Add Prefix and Postfix Increment Overflow and Decrement Underflow Cases 5

2.2 Missing BOF 6

2.3 Unintended BOF/Read/Above for 64-bit Architectures 7

2.4 Check for Allocation Failure 8

2.5 BOF/Stack Accessing Memory After Its Lifetime 10

2.6 Memory Accessed After Its Lifetime 11

2.7 Uninitialized Structure Member 11

2.8 Undefined Behavior in Random Number Macros 12

2.9 No Unix Command Injection 13

2.10 Insecure Temporary Files $\quad 14$

2.11 Wrong Check for Value Out of Range - int 15

2.12 Wrong Check for Value Out of Range - int64_t 17

2.13 Wrong Check for Value Out of Range - unsigned int 18

2.14 Wide Format Strings Mishandled 18

2.15 Wrong fscanf() Format Specifier for int64_t and size_t 19

2.16 Improve Compile Files and Scripts 20

3 Known Problems in Juliet 1.3

3.1 Memory Leaks 21

3.2 No Evident Failure $\quad 22$

3.3 Memory Accessed After Its Lifetime 22

3.4 Check for Value Out of Range Still Wrong - int64_t 23

3.5 Additional Cases with Wrong Check for Value Out of Range 23

3.6 Wrong Format Specifier for Wide String 24

3.7 Wrong Format Specifier to Print char as Hexadecimal 24

3.8 Many Bugs Removed by Using Hardcoded Values 25

3.9 Uncaught Java Exceptions 26

3.10 Dead Stores 26

3.11 Dead Code not in Metadata 27

3.12 Integer Overflow not in Metadata 28

3.13 Temporary Files Still Not Secure 28

3.14 Suggestions We Did Not Take 28

4 Some Thoughts on the Future of Juliet and Test Suites 29

References $\quad 30$ 


\section{Change Log}

August 2019

Corrected the subdirectories where we placed the new prefix increment and decrement cases. Section 2.1. 


\section{Introduction}

We are pleased to announce Juliet 1.3, which was released in October 2017.

Juliet 1.3 consists of tens of thousands of small test programs in $\mathrm{C} / \mathrm{C}++$ and Java exhibiting over 100 classes of errors. It replaces Juliet 1.2. The $\mathrm{C} / \mathrm{C}++$ part contains 64099 test cases and more than 100000 files. The Java part contains 28886 test cases and more than 46000 files. Both parts also include files, scripts, headers, and other material for compiling the test cases, either as a single program per test case or as one program of all test cases in a given language. These cases should be useful in Unix, Microsoft Windows, and other environments. The Juliet test suite was originally developed by the National Security Agency's Center for Assured Software (CAS) and was first released in December 2010. We now refer to it as Juliet Version 1.0.

The $\mathrm{C} / \mathrm{C}++$ part of Juliet 1.0 comprised 45324 test cases [1] covering 116 Common Weakness Enumeration (CWE) entries [2], and the Java part comprised 13801 cases [3] covering 106 CWEs. The following year, Version 1.1 added a few additional CWEs and increased the total number of test cases to 81056 . To add methods for building test cases, Version 1.1.1 was released for the Java part.

Version 1.2 was released in May 2013 with a total of 86864 test cases. A dozen CWEs were added, and during quality control review, CAS determined that test cases for the CWEs listed in Table 1 were invalid and removed them from the Java part [4].

Table 1. CWEs removed from the Java part of Version 1.2.

\begin{tabular}{|c|l|}
\hline CWE & Name \\
\hline 180 & Incorrect Behavior Order: Validate Before Canonicalize \\
\hline 330 & Use of Insufficiently Random Values \\
\hline 489 & Leftover Debug Code \\
\hline 497 & Exposure of System Data to an Unauthorized Control Sphere \\
\hline 514 & Covert Channel \\
\hline 547 & Use of Hard-coded, Security-relevant Constants \\
\hline 665 & Improper Initialization \\
\hline 784 & $\begin{array}{l}\text { Reliance on Cookies without Validation and Integrity Checking } \\
\text { in a Security Decision }\end{array}$ \\
\hline
\end{tabular}

Similarly, Table 2 lists the CWEs that CAS determined were invalid and removed from the $\mathrm{C} / \mathrm{C}++$ part [5]. These are still available from the Software Assurance Reference Dataset (SARD) Test Suites page [6, 7] in Juliet Version 1.1. 
Table 2. CWEs removed from the $\mathrm{C} / \mathrm{C}++$ part of Version 1.2.

\begin{tabular}{|c|l|}
\hline CWE & Name \\
\hline 204 & Response Discrepancy Information Exposure \\
\hline 304 & Missing Critical Step in Authentication \\
\hline 374 & Passing Mutable Objects to an Untrusted Method \\
\hline 392 & Missing Report of Error Condition \\
\hline 489 & Leftover Debug Code \\
\hline 547 & Use of Hard-coded, Security-relevant Constants \\
\hline 560 & Use of umask() with chmod-style Argument \\
\hline
\end{tabular}

Flow variant 19, dead code after a return, was removed to reduce incidental dead code. (See the next section for an explanation of flow variants.) Two flow variants were added: 83, declaring class objects on the stack, and 84, declaring them in the heap. In addition, directories with many files were split into smaller subdirectories, so that no directory had more than 1000 files.

In the years since Version 1.2 was released, people using it reported unintentional problems they found and passed along suggestions for improvement. We received particularly extensive comments from Pascal Cuoq and André Maroneze. In 2016 one NIST researcher, Eric Trapnell collected much external and internal feedback and many notes, and we began to create a new version of Juliet to address the problems.

Juliet 1.3 fixes about two dozen systematic problems in Version 1.2. The fixes changed 21552 files.

This technical note details the changes from Version 1.2. The next section, 1.1, briefly explains how the thousands of test cases in Juliet are organized, the case naming scheme, and the structure of each case. Section 1.2 is a very brief description of the fixes and changes. They are listed roughly in decreasing importance. Section 2 details each fix or change. In spite of all the changes, we know of many problems remaining in Juliet 1.3. Section 3 lists them and also lists suggestions that we did not take. Finally, Sec. 4 offers some thoughts about the future of Juliet and test suites in general.

\subsection{The Organization of the Juliet Suite of Test Cases}

The Juliet suite of test cases consists of two parts: test cases and supporting files for Java and test cases and supporting files for $\mathrm{C}$ and $\mathrm{C}++$. Each part is available in two forms: a complete, structured, stand-alone suite and a suite of individual cases. These are available from the Software Assurance Reference Dataset (SARD) Test Suites page [6, 7].

The stand-alone suites include the CAS documentation for Version 1.2, shared support code and "include" files, means to compile the test cases (and scripts to update them if one adds or removes cases), and input files. Each CWE has its own subdirectory, e.g., CWE338_Weak_PRNG or CWE764_Multiple_Locks. CWEs with fewer than one thousand test case files contain all their test cases directly under its subdirectory. For CWEs with more 
than one thousand files, the test cases are divided into subdirectories named s01, s02, etc. There is more information in the $\mathrm{C} / \mathrm{C}++$ or Java User Guides $[4,5]$.

In this document, we usually refer to just the CWE number, instead of the complete directory name, which includes the CWE name.

We use the Bugs Framework (BF) [8] in many instances for more clear and precise classification than is possible with CWEs.

Every Juliet test case is available as a separate test case in the SARD, with its own SARD ID number. For instance, CWE80_XSS__Servlet_getParameter_Servlet_03.java is 145277, and CWE457_Use_of_Uninitialized_Variable__double_pointer_15.c is 240543. The suites of individual cases, SARD test suites 108 and 109, organize test cases by their SARD ID number. Each test case has its own subdirectory. The subdirectories are organized by the millions digits, then thousands digits, then units digits. For example, the path to the first test case is $000 / 145 / 277 /$, and the path to the second is $000 / 240 / 543 /$.

Many of the cases were not changed from Version 1.2. If the case was not changed, the Version 1.2 case is used and the SARD ID number remains. If the case was changed, we deprecated the 1.2 case in the SARD and added a new case to the SARD.

Each test case has a unique file name. The file name consists of the CWE number and name, two underscores (_-), followed by various identifying types, functions, and alternatives, then a control flow variant number. Control flow variant numbers are the same across the entire Juliet suite. For instance, _03 variants wrap the target code in a conditional: if $(5==5)$.

Most test cases consist of a single file, but some span multiple files. Those with multiple files use a one-letter suffix. For instance, 77913 consists of four files:

CWE127_Buffer_Underread__malloc_char_memmove_53a.c, CWE127_Buffer_Underread__malloc_char_memmove_53b.c,

CWE127_Buffer_Underread__malloc_char_memmove_53c.c, and

CWE127_Buffer_Underread__malloc_char_memmove_53d.c.

Instead of a one-letter suffix, some Java test cases use other suffixes, e.g., _bad, _base, or _goodG2B.

Additional information can be found in Boland and Black [9].

Each test case has a particular structure. Each has a single function intended to manifest a bug and has one or more functions with similar behavior, but with no bug. In this document we refer to the buggy code as bad code and the bug-free code as good code.

Problems were reported in both bad code and good code. Some code in Version 1.2 did not have the intended bug, or it had unintentional bugs. Typically, we show a bit of the code from Version 1.2, which we refer to as old code, and the corresponding bit from Version 1.3 , which we refer to as new code. 


\subsection{Summary of Changes and Remaining Issues}

This section summarizes the changes made to Juliet Version 1.2 to create Version 1.3. Section 2 details each change. This section also summarizes the problems that we know are still in Version 1.3. Section 3 details the problems and issues.

Version 1.2 had no test cases of integer overflow using unary increment ( $i++$ and $++i$ ) operators or test cases of integer underflow using decrement ( $i--$ and $--i$ ) operators. We created 3404 Java test cases (5612 files) and $2736 \mathrm{C}$ test cases (4032 files) to manifest overflow or underflow. We added overflow cases under CWE190 and underflow cases under CWE191. For details, see Sec. 2.1.

- Fixed $104 \mathrm{C}$ cases to actually have buffer overflow (BOF) [10, 11]. Also fixed CWE121 Stack-based Buffer Overflow cases to allocate on the stack (Sec. 2.2).

- Fixed $144 \mathrm{C}$ cases that had unintended BOF/Read/Above from constant strings in 64-bit architectures (Sec. 2.3).

- Added a simple check for allocation failure (NULL pointer) to $11619 \mathrm{C}$ files across 20 CWEs (Sec. 2.4).

- Removed 24 C BOF/Stack cases (51 files) under CWE121 that allocated memory on the stack in a subfunction, then used it after its lifetime-after return. We could not identify a way to fix the cases and still fulfill their test purposes (Sec. 2.5).

- Fixed $168 \mathrm{C}$ files to not access memory after its lifetime (Sec. 2.6).

- Fixed 294 C files to initialize both members of a structure (Sec. 2.7).

- Fixed the $\mathrm{C}$ random number macros so their behavior was well defined (Sec. 2.8).

- Fixed $5200 \mathrm{C}$ test cases (8120 files) under CWE078 to have OS injection on Unix (Sec. 2.9).

- Improved $72 \mathrm{C}$ files to use mkstemp() as a more secure way to create temporary files (Sec. 2.10).

- Fixed $610 \mathrm{C}$ files in 576 cases to correctly guard against possible overflow. Because the problems and fixes differ for different types, we detail changes for int type cases (Sec. 2.11), for int64_t cases (Sec. 2.12), and for unsigned int cases (Sec. 2.13) separately.

- Fixed 672 files to use swprintf instead of snprintf () to handle wide character string formats (Sec. 2.14).

The following changes did not invalidate the test cases, that is, not serve as a test for the intended bug, or add unintentional serious bugs. However, they improved Juliet and were worth making.

- Fixed code to use the correct format specifier in $f \operatorname{scanf}()$ for variables of type int64_t (352 files) and size_t (200 files). Also fixed the utility file io.c to use the correct format specifiers for those types. In addition, changed io.c to include files to properly declare macros (Sec. 2.15).

- Changed the compile ("make") process to be far more efficient. Also made other improvements and clean-ups (Sec. 2.16). 
The astute reader may wonder why there were so many changes to $\mathrm{C}$ cases and not many to Java cases. We propose several reasons. First, memory allocation in $\mathrm{C}$ is very prone to errors, and most such errors cannot occur in Java. Second, $\mathrm{C}$ is an older language with many nuances about format and types that caused problems. Third, some problems that could have been present in the Java cases, such as wrong check for value out of range (Sec. 2.11), were not present; the code was correct in the earliest version.

Juliet Version 1.3 contains numerous changes from Version 1.2. However, many issues remain in Version 1.3. These are detailed in Sec. 3. Here is a summary of each issue. Thousands of cases have minor memory leaks (Sec. 3.1). Many cases have faults regardless of the input or do not exhibit failure at all (Sec. 3.2). Hundreds of cases access memory after its lifetime (Sec. 3.3) or have out-of-range checks that are still wrong (Sec. 3.4 and Sec. 3.5). A few cases have the wrong format specifier for wide strings (Sec. 3.6) or unintentional dead stores (Sec. 3.10). A utility function incorrectly prints the value 255 (Sec. 3.7). Many intentional bugs are removed in the good code by using a hardcoded value (Sec. 3.8). Some Java cases potentially leak stream resources (Sec. 3.9). There is no metadata indicating thousands of instances of dead code (Sec. 3.11) or hundreds of intentional integer overflows (Sec. 3.12). Temporary file names are still not fully secure (Sec. 3.13).

\section{Details of All Changes}

This section details each change to Version 1.2. The amount and kind of comment or description differs for each problem. For instance, some problems include an exhausting explanation of exactly why something is a bug. Others include how we gained assurance that all instances of a mistake were fixed or that there were no unintentional changes.

We provide the number of test cases or files associated with each change, usually listing them for future review. When the names of the files follow a certain pattern, we give the pattern using shell file name completion "star" $(*)$ notation.

We usually edit the code that we include for examples to make it fit the printed page and to eliminate superfluous parts, so the reader may grasp the essentials more easily. Complete code is always available from the SARD.

Several people pointed out problems or made suggestions over the years following the release of Version 1.2. For attribution and historical purposes, most changes include a few words on the source and a convenient designation. Pascal Cuoq designated his comments with letters. André Maroneze used numbers. Eric Trapnell collected many comments and suggestions, and we tracked some of our work by row number in his spreadsheet.

\subsection{Add Prefix and Postfix Increment Overflow and Decrement Underflow Cases}

While tracking down a bug in a project that used Juliet, we realized that there were no test cases of integer overflow involving a prefix increment $(++i)$ or postfix increment $(i++)$ operator or of underflow involving a prefix decrement $(--i)$ or postfix decrement (i--) operator. 
There were cases for overflow and underflow for other operations, such as multiplication or addition:

$$
\text { int result }=\text { data }+1 \text {; }
$$

For C, we created prefix increment overflow cases from CWE190 *_add_*.c cases, named them *_preinc_*. c, and placed them under CWE190 in a new subdirectory, s07. Prefix increment cases were a straight-forward syntactic substitution. The postfix increment cases were more subtle since the variable value changes after the value is retrieved. One alternative is to use the comma operator to put everything on one line:

$$
\text { int result }=(\text { data }++ \text {, data }) \text {; }
$$

However, we thought this construct was too unusual, so we added another line with the actual increment:

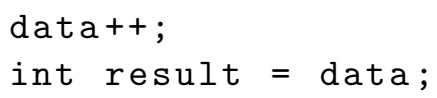

We created postfix increment overflow cases from the prefix increment cases, and named them *_postinc_*. c. We placed them in another new subdirectory, s06.

The cases of underflow from the prefix and postfix decrement operators were analogous. We began with CWE191 *_sub_*.c cases and placed the new cases under CWE191, _postdec_in s04 and _predec_ in s05.

Java cases came from CWE190 *_add_*.java and CWE191 *_sub_*.java. We placed them under CWE190, _postinc_ in s06 and _preinc_in s07, and CWE191, _postdec_ in s04 and_predec_in s05.

This added 684 test cases (1008 files) in each new C subdirectory and 851 cases (1403 files) in each new Java subdirectory, for a total of 2736 C cases (4032 files) and 3404 Java cases (5612 files).

\subsection{Missing BOF}

We use attributes of the Bugs Framework [10] Buffer Overflow (BOF) [11] class to classify bugs that are variously referred to as buffer overflow, out-of-bounds read, incorrect access of indexable resource, etc. These attributes are orthogonal and include access (either read or write), boundary (below/before or above/after), and location (heap, stack, etc.). Hence the title of this section may be read as "Missing Buffer Overflow."

There are $96 \mathrm{C}$ cases (104 files) under CWE122 s06 and CWE121 s01 that test the misuse of strlen() with wide character strings, which is CWE-135. As written, they did not cause buffer overflow. The following example of old code is from CWE122_Heap_Based_ Buffer_Overflow__CWE135_01.c, which is deprecated 70400. (We note "deprecated" since it may not appear in default SARD searches.)

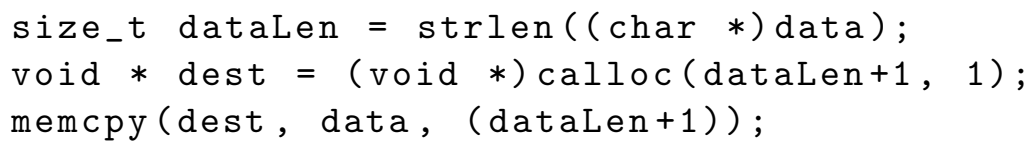


It has several problems. Note that data is a wide string. That was the intended bug; strlen() stops too early on wide strings and gives an incorrectly short length. dest was intended to be too small because the length is short. It is also too small because only one byte per character is allocated. However, using memcpy() prevents any buffer overflow! It copies the same number of bytes that were allocated.

The following example of new code is from CWE122_Heap_Based_Buffer_Overflow_CWE135_01.c 232119:

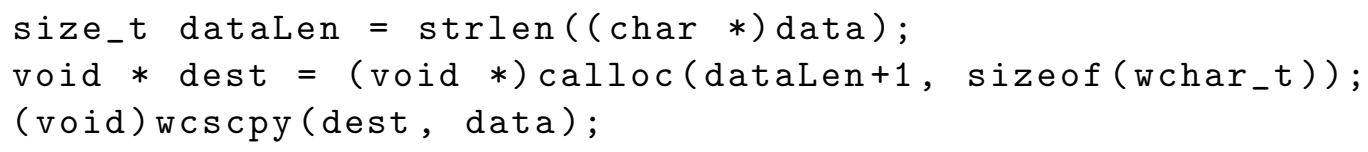

In the new code, calloc() allocates wide characters (but still not enough). More importantly, wcscpy () copies the whole wide string, which is BOF/write [11].

While working on this problem, we noticed that CWE-121 is Stack-based buffer overflow, but the buffer is allocated in the heap. We changed those cases to use alloca(), via a macro, instead of calloc (). The following example is from CWE121_Stack_Based_Buffer_ Overflow__CWE135_01.c 231402.

void *dest $=(\operatorname{void} *) \operatorname{ALLOCA}\left(\left(\right.\right.$ dataLen $\left._{1}\right) * \operatorname{sizeof}($ wchar_t $\left.)\right)$;

To correct a different problem, we also added code to check if the allocation succeeds. See Sec. 2.4 for details.

All of the test cases had __CWE135_ in their names. We deprecated and replaced SARD IDs 62948 to 62995 and 70400 to 70447.

Pascal Cuoq reported this problem on 25 June 2013 (comment C), Takashi Matsuoka reported it on 22 August 2013, and André Maroneze reported it on 12 June 2017 (suggestion 7). This problem was Eric Trapnell's rows 2 and 12.

\subsection{Unintended BOF/Read/Above for 64-bit Architectures}

In 64-bit architectures, $144 \mathrm{C}$ cases have unintended BOF/Read/Above [11] from constant strings. The following old code is from CWE121_Stack_Based_Buffer_Overflow__char_ type_overrun_memcpy_01.c deprecated 63036:

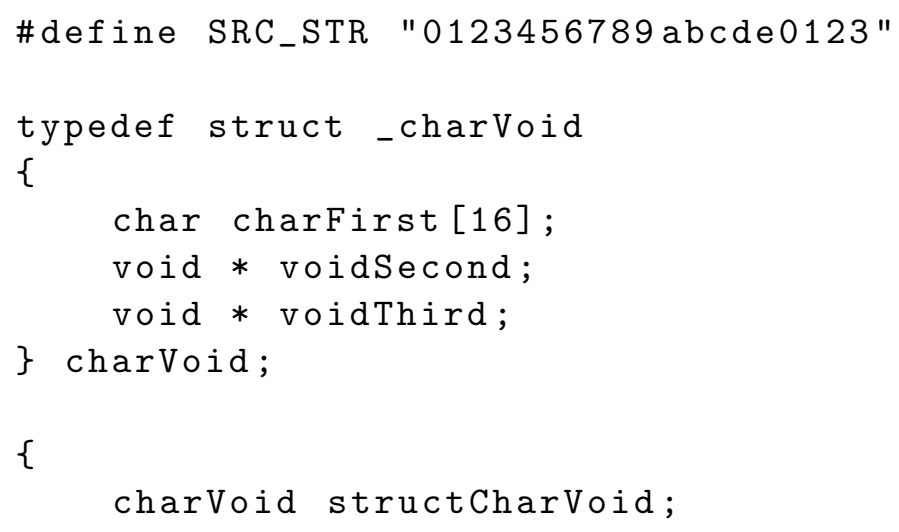




$$
\begin{aligned}
& \text { memcpy (structCharVoid.charFirst, } \\
& \text { SRC_STR, } \operatorname{sizeof(structCharVoid));~}
\end{aligned}
$$

The string SRC_STR is 20 characters long, including the NULL. A 64-bit architecture could have 64 -bit ( 8 byte) pointers. charVoid is then $16+8+8=32$ bytes, so the old code read 32 characters from a 20 character string. To fix this, we extended the string to 32 characters:

\#define SRC_STR "0123456789abcdef0123456789abcde"

There are 36 cases under each of CWE121 s01 and CWE122 s01 (18 char_*_memcpy cases and 18 char_*_memmove cases respectively), and 36 under each of CWE121 s09 and CWE122 s09 (also 18 char_*_memcpy cases and 18 char_*_memmove cases). We deprecated and replaced SARD cases 63036 to 63071 and 67448 to 67483.

Pascal Cuoq explained this problem in his email of 19 March 2014. This problem was Eric Trapnell's rows 5 and 6.

\subsection{Check for Allocation Failure}

Thousands of $\mathrm{C}$ cases allocated memory. Few checked whether the allocation succeeded or failed. They just used the memory. Here is an example from CWE401_Memory_Leak__struct _twoIntsStruct_malloc_01.c deprecated 100474:

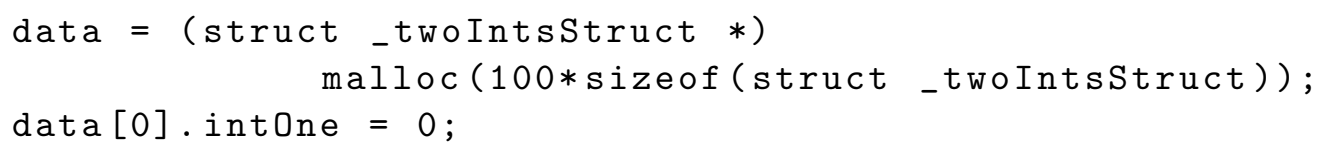

If the allocation fails, a NULL pointer is returned. Dereferencing a NULL pointer causes an undefined state. In the C language, "undefined" is more drastic than "the result may be any number." It means that following the dereference of a NULL pointer, "the program can whistle 'Happy Birthday' in all the colors of the rainbow and still be considered to conform to the standard." [12]

In particular, Frama-C halted analysis at the second statement above. After that, anything is allowed, so no precise analysis is reasonable. For the Static Analysis Tool Exposition (SATE) V Ockham Sound Analysis Criteria [12], Frama-C was run twice: once with allocation modeled as always succeeding, and once with allocation modeled as failing. The union of those two runs served the intended purposes.

We added simple checks after malloc(), calloc(), or realloc(). The check was minimal, as shown in the new version, 239815, which replaced the former case:

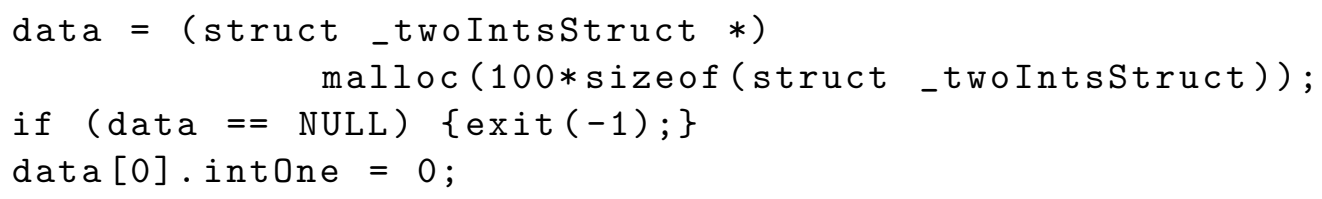

This check enables sound tools to continue analysis, since the state is always well defined.

We changed 11619 files under 20 CWEs. We do not detail the files changed. Table 3 summarizes the number of files and test cases changed under each CWE. 
Table 3. Number of files and test cases under each CWE directory to which we added checks for allocation failure.

$\begin{array}{rrl}\begin{array}{l}\text { Number } \\ \text { of files }\end{array} & \begin{array}{r}\text { Number } \\ \text { of cases }\end{array} & \text { CWE subdirectory name } \\ 4 & 4 & \text { CWE121_Stack_Based_Buffer_Overflow } \\ 3342 & 3186 & \text { CWE122_Heap_Based_Buffer_Overflow } \\ 500 & 480 & \text { CWE124_Buffer_Underwrite } \\ 300 & 288 & \text { CWE126_Buffer_Overread } \\ 500 & 480 & \text { CWE127_Buffer_Underread } \\ 312 & 288 & \text { CWE194_Unexpected_Sign_Extension } \\ 312 & 288 & \text { CWE195_Signed_to_Unsigned_Conversion_Error } \\ 72 & 72 & \text { CWE244_Heap_Inspection } \\ 18 & 18 & \text { CWE364_Signal_Handler_Race_Condition } \\ 1008 & 972 & \text { CWE401_Memory_Leak } \\ 312 & 288 & \text { CWE415_Double_Free } \\ 150 & 150 & \text { CWE416_Use_After_Free } \\ 251 & 242 & \text { CWE457_Use_of_Uninitialized_Variable } \\ 54 & 54 & \text { CWE467_Use_of_sizeof_on_Pointer_Type } \\ 18 & 18 & \text { CWE479_Signal_Handler_Use_of_Non_Reentrant_Function } \\ 312 & 288 & \text { CWE680_Integer_Overflow_to_Buffer_Overflow } \\ 234 & 234 & \text { CWE758_Undefined_Behavior } \\ 576 & 576 & \text { CWE761_Free_Pointer_Not_at_Start_of_Buffer } \\ 2784 & 2694 & \text { CWE762_Mismatched_Memory_Management_Routines } \\ 560 & 480 & \text { CWE789_Uncontrolled_Mem_Alloc }\end{array}$

Some test cases intentionally have possible NULL pointer dereference. We did not change them.

Eighteen cases under CWE476 intentionally omit checks immediately after allocation in both bad and good code. These cases check whether an analyzer will warn about a NULL check after a dereference. If a pointer is NULL and is dereferenced, the program has trouble. Any NULL check after that is of little use. The following example code is from CWE476_NULL_Pointer_Dereference__null_check_after_deref_01.c 104778:

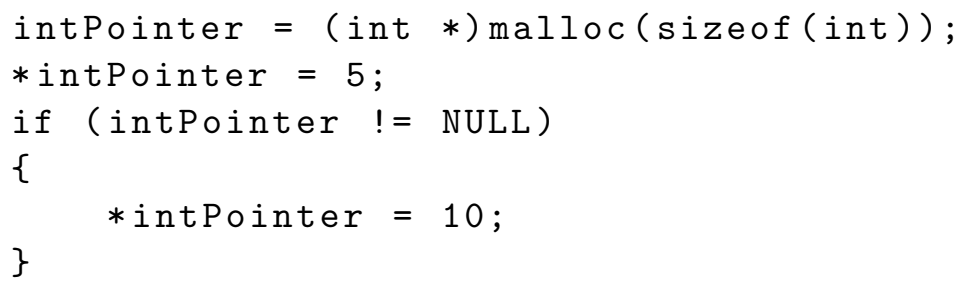

This situation may arise in code if at one time there was a check immediately after the allocation, but later maintenance added the dereference before the check. Another possibility is that the code is in a context where the reference is never NULL, i.e., it was checked much earlier. A NULL check following a dereference suggests sloppy code at best.

The bad code of many cases under CWE690 does not check for NULL, while the 
good code checks for NULL. The following example code is from CWE690_NULL_Deref_ From_Return__fopen_01.c 111122:

data $=$ fopen ("file.txt", "w+");

fclose (data);

If fopen() fails, it returns NULL.

As with the other changes, we used small scripts to modify the files. Since we changed so many files and there were so many different circumstances, we made several special checks to verify the edits. In particular, we did not want to add checks that would remove the intended bug and invalidate the test case. We manually reviewed dozens of files, whilst developing and testing the editing script. After editing, we checked that the number of allocations and the number of NULL checks matched. We carefully audited the mismatches. We also compiled every test case individually as a simple check of correctness.

Pascal Cuoq reported this problem on 22 July 2013 (comment M). André Maroneze reported it on 12 June 2017 (suggestion 4). This problem was Eric Trapnell's row 13.

A related problem is thousands of "memory leaks," i.e., memory is allocated, but never freed. We saw little benefit and great cost to fix this problem, so we did not deal with it. We provide details in Sec. 3.1.

\subsection{BOF/Stack Accessing Memory After Its Lifetime}

We removed $24 \mathrm{C}$ cases (51 files) that allocated memory on the stack, then used the memory after its lifetime. The following example code is from CWE121_Stack_Based_Buffer_ Overflow__CWE131_memcpy_21.c 62870:

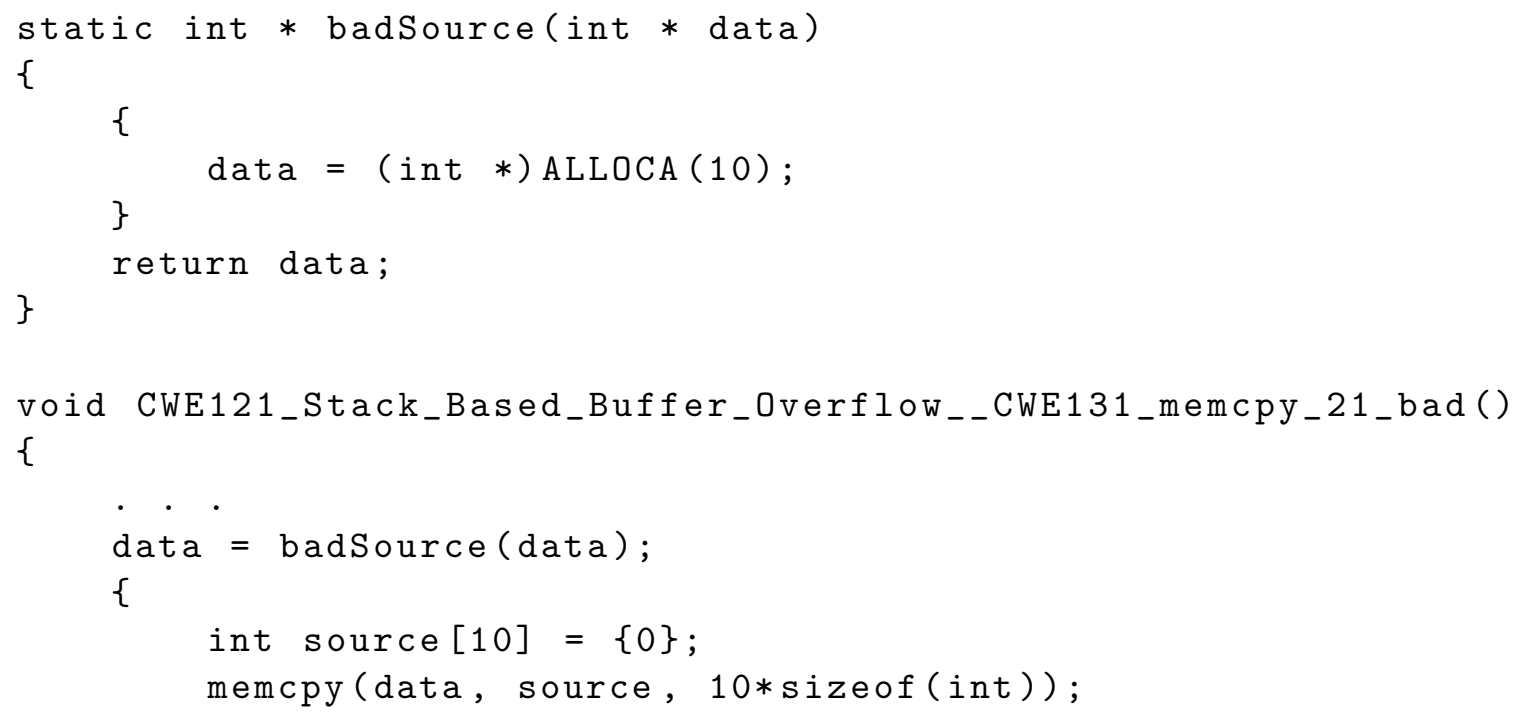

ALLOCA() is a macro for alloca(), which is not defined in C11 or POSIX. It allocates memory on the stack. In typical implementations, the end of badSource() will terminate the memory's lifetime. 
These cases are under CWE121 s01. They are CWE121*_CWE131_ functions loop, memcpy, and memmove, and variants $21,22,42,43,61,62,83$, and 84 . The purpose of these tests is to allocate memory in one function, then use the memory in another. We could not identify a way to fix these cases and fulfill their test purposes, so we removed them.

Bertrand Stivalet reported this problem on 10 March 2014. This problem was Eric Trapnell's row 8.

\subsection{Memory Accessed After Its Lifetime}

Cases with a total of $168 \mathrm{C}$ files under CWE476 accessed automatically allocated memory after its lifetime. The following example good code is from CWE476_NULL_Pointer_ Dereference_-_int64_t_01.c deprecated 104652:

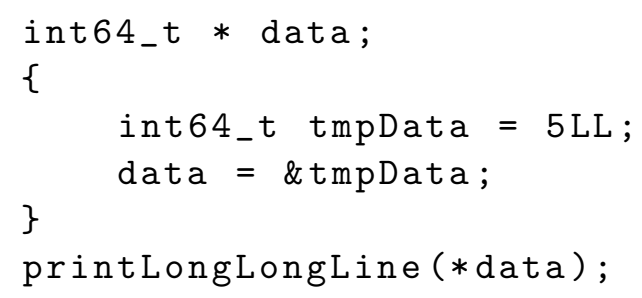

Memory for tmpData is automatically allocated (usually on the stack) when execution enters the block. Although the address is saved, the lifetime of the memory ends when execution leaves the block. Thus, the argument of the call to printLongLongLine () is an invalid dereference.

We fixed this by declaring the variable earlier, as shown in the new good code 240719 :

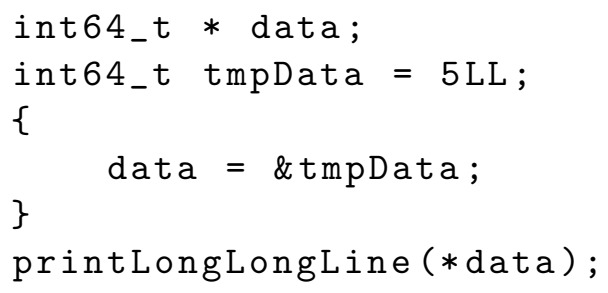

Pascal Cuoq reported this problem on 26 June 2013 (comment G). André Maroneze reported this problem on 12 June 2017 (suggestion 5). This problem was Eric Trapnell's row 10.

After Juliet 1.3 was released, André Maroneze reported another set of cases that accessed memory after its lifetime. We provide details in Sec. 3.3.

\subsection{Uninitialized Structure Member}

While tracking down what we thought were incorrect warnings of uninitialized variables for the SATE V Ockham Sound Analysis Criteria, we found a wide-spread problem. One type of structure has two members: 


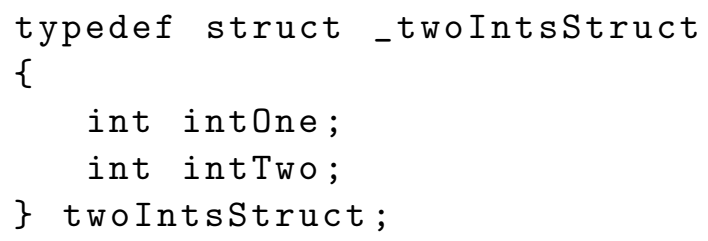

In 1046 files, both fields are initialized:

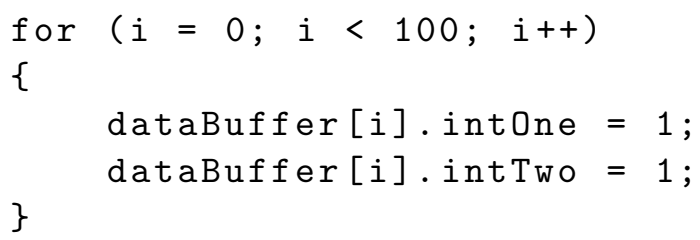

However, in 294 files, intOne is initialized twice, and intTwo is not initialized:

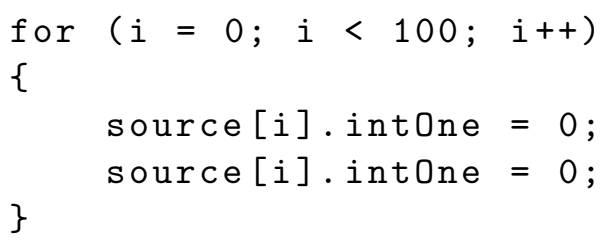

We believe this was unintentional. We changed 240 files under CWE121 s04 and s05 (the files are named *_CWE805_*) and under CWE476. We deprecated and replaced test cases 64792 to 65031 .

Pascal Cuoq reported this problem on 26 June 2013 (comment H). André Maroneze reported this problem on 12 June 2017 (suggestion 6). This problem was Eric Trapnell's row 9.

\subsection{Undefined Behavior in Random Number Macros}

The behavior of macros that Juliet 1.2 used for random numbers, RAND32 and RAND64, were undefined. The macros shift signed integers out of range. The following old code is from testcasesupport/std_testcase.h:

\#define RAND32 ()$\quad((\operatorname{rand}()<<30)-(\operatorname{rand}()<<15)$ - $r$ and ()$)$

\#define RAND64 ()$((\operatorname{rand}()<<60)-(\operatorname{rand}()<<45)-(\operatorname{rand}()<<30)$

- $(r$ and ()$<<15)$ rand ()$)$

As explained in Sec. 2.4, Frama-C ceased analysis after this undefined behavior.

We replaced the macros following André Maroneze's suggestions. The shifts are done on unsigned integers so that the behavior is defined. A single call to rand() then picks whether to produce a positive or a negative number. The new code for RAND32 is the following:

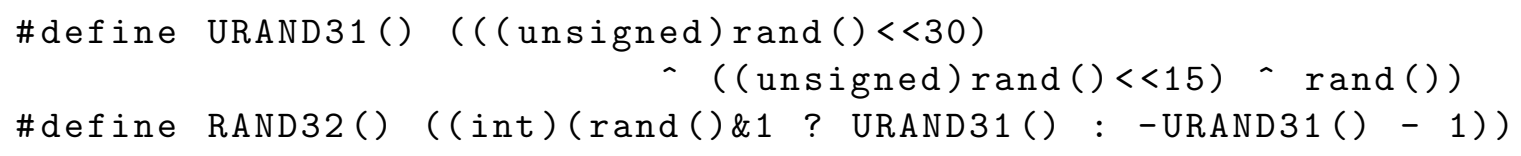


Note that this does not guarantee numbers with very high entropy. Specifically, there are few guarantees for numbers that rand() produces. There are far better ways to generate random numbers, but this seems sufficient for Juliet. We tested both of the new macros with an extensive pseudo-random number generator test program, and they both passed.

Although we only changed one file, this corrected undefined behavior in $3440 \mathrm{C}$ test cases under 16 CWE directories.

Java cases in Juliet use random booleans for conditionals, but do not use "large" random numbers.

André Maroneze reported this problem on 12 June 2017 (suggestion 1).

\subsection{No Unix Command Injection}

The INJ [13] test cases, under CWE078, did not work in Unix platforms. The code compiles and executes, but does not perform a command injection. The following example of the old code is from CWE78_OS_Command_Injection_-char_console_execl_41.c deprecated 118447:

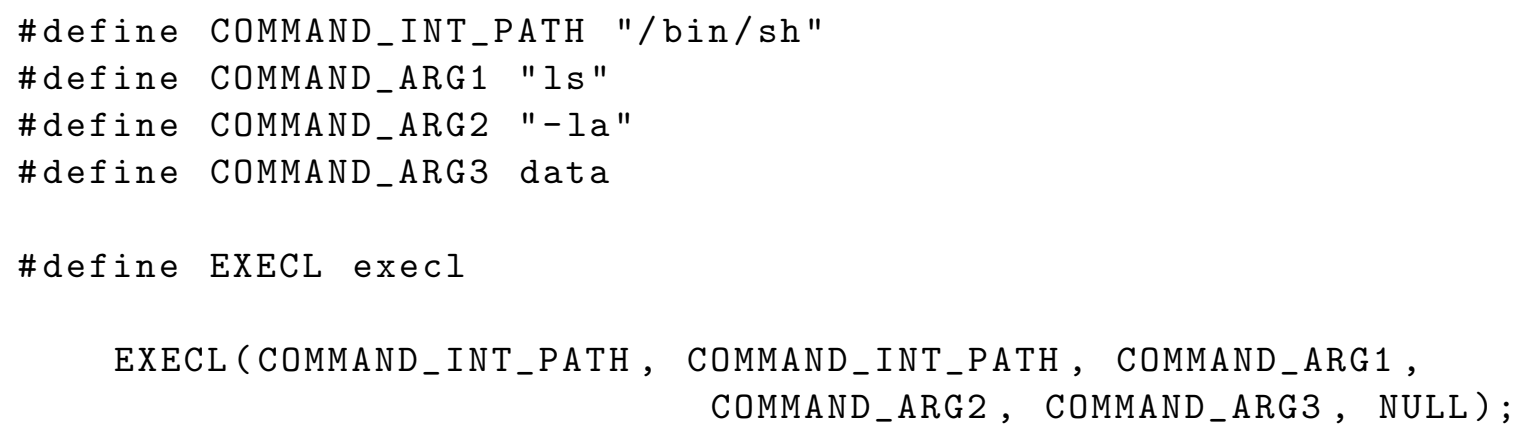

The program reads a string into data then executes the EXECL() statement. Suppose that the input is $*$; date>GOTCHA. The program executes this command:

/bin/sh ls -la *; date >GOTCHA

This is not valid: sh does not execute binaries. It "treats the first argument as the name of a file from which to read commands (a shell script)" [14].

Charles De Oliveira developed the following code, which works in Windows and in Unix. He changed the execution statement and two macro definitions. The following new code is from CWE78_OS_Command_Injection__char_console_execl_41.c 244499:

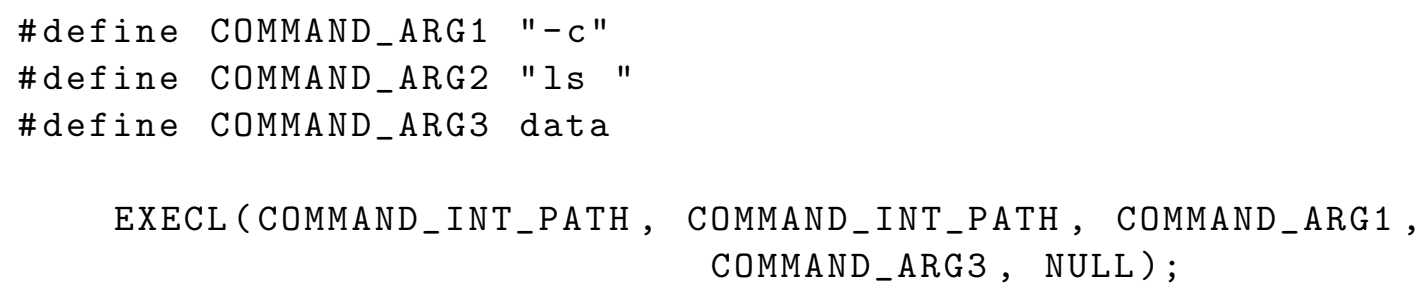

The program reads a string, appends it to ls (in code not shown), and puts it into data. If the input is again *; date>GOTCHA, the program executes this command: 
/bin/sh -c ls *; date >GOTCHA

After executing $1 \mathrm{~s}$, this executes date and puts the output in a local file.

In the 520 files that use popen(), we also corrected the argument to popen(). The following example old code is from CWE78_OS_Command_Injection__char_file_popen_01.c deprecated 119479:

$$
\text { pipe = POPEN (data, "wb"); }
$$

The argument wb is not POSIX standard [15].

The following new code is from 245515:

$$
\text { pipe = POPEN (data, "w"); }
$$

We changed $5600 \mathrm{C}$ files, $2120 \mathrm{C}++$ files, and 400 header (.h) files, totaling 8120 files, in 4800 test cases under CWE78. There were 840 files in each of eight subdirectories, s01 through s08, and 280 files in s09.

Elisa Heymann reported this problem on 1 October 2014. This problem was Eric Trapnell's row 7.

\subsection{Insecure Temporary Files}

Test cases under CWE377 intentionally insecurely create and open temporary files. The bad functions used tempnam(), tmpnam(), or mktemp(). The following bad code is from CWE377_Insecure_Temporary_File__char_mktemp_01.c deprecated 97938:

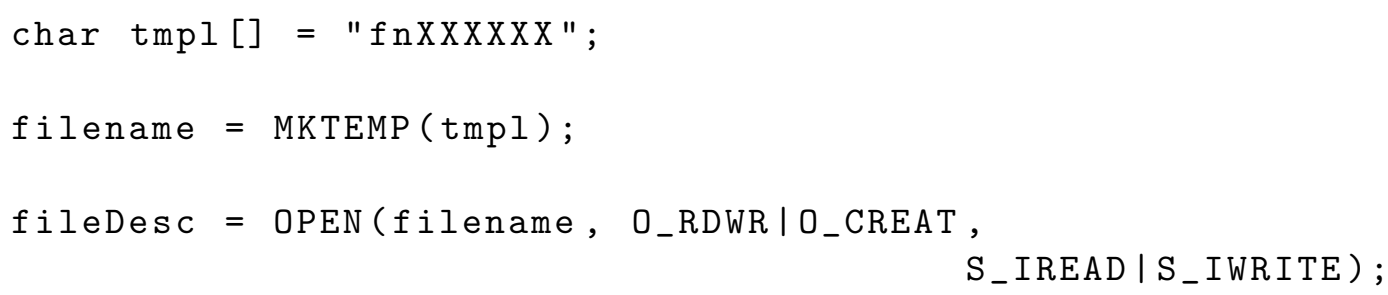

The old good functions were more secure than the old bad functions: they opened files with O_EXCL. The following old good code to open the file is from the same case as above:

$$
\begin{array}{r}
\text { fileDesc }=\text { OPEN(filename, O_RDWR|O_CREATIO_EXCL, } \\
\text { S_IREAD|S_IWRITE); }
\end{array}
$$

Using mkstemp() makes the code more secure still. (However, file names are still too predictable for this to be considered very secure, but it is better. We repeat this explanation in Sec. 3.13 for consistency.) The following new good code is from 239333:

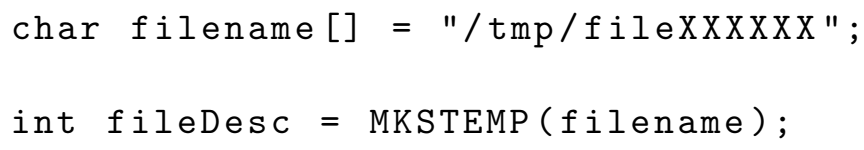

This change potentially alters how the cases can be used. The cases now use a more secure function; they do not just use a dangerous function more securely. This raises the possibility that these cases might serve, also or instead, for CWE-242 Use of Inherently 
Dangerous Function. Currently the test cases under CWE242 only have variants of one function, gets (). We decided that since Juliet does not include some number of dangerous functions, there was no reason to take time using these cases there.

In checking for other possible uses, we found a problem in test cases under CWE459, which deals with incomplete cleanup after execution. The test cases do not remove a temporary file. The following example old bad code is from CWE459_Incomplete_Cleanup_-char _01. c deprecated 104285:

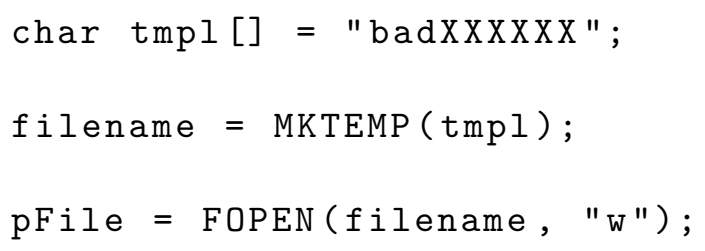

The good code is the same, except that the prefix of the file's name is "good" instead of "bad." To remove the unintentional bug, we changed these cases to use mkstemp(), too. The following new good code is from 240619:

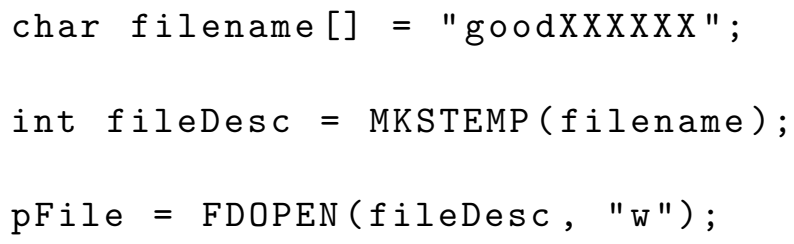

To compile in Windows, Eric Trapnell found an open source version of mkstemp(), which we added to each file.

We did not change any wchar_t cases. In those cases, the file name is declared as

wchar_t *filename;

No Unix version of mkstemp() handles wchar_t, and Windows does not have mkstemp().

We changed 54 files under CWE377 to use mkstemp() in the good functions. They were named CWE377_Insecure_Temporary_File___char_FUNC with 18 files where FUNC is tempnam, 18 tmpnam, and 18 mktemp. We also changed 18 files under CWE459 to use mkstemp(). They were named CWE459_Incomplete_Cleanup__char_01.c to _18.c.

André Maroneze reported this problem on 12 June 2017 (suggestion 8). This problem was Eric Trapnell's row 11.

\subsection{Wrong Check for Value Out of Range - int}

Some of the good code under CWE190_Integer_Overflow intended to avoid overflow by checking that the value was in range. The following example of good code is from CWE190_ Integer_Overflow_-int_fscanf_square_01.c deprecated 83358:

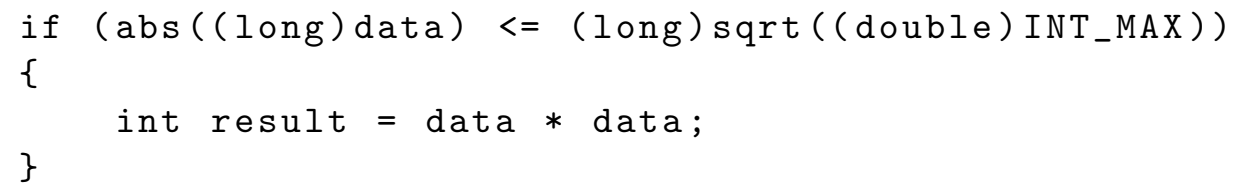




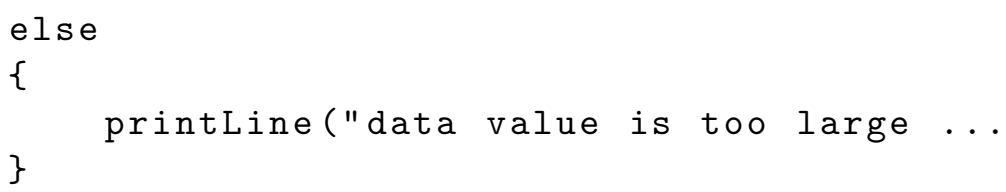

The problem is that the absolute value of the most negative integer (abs (INT_MIN)) is undefined because it is greater than INT_MAX. Some systems define abs (INT_MIN) to return INT_MIN, which is negative. In that case, it would pass the test anyway and cause an overflow. In addition, the function abs () does not handle long values; at best the cast is not needed. The following example of the check in new good code is from 235621:

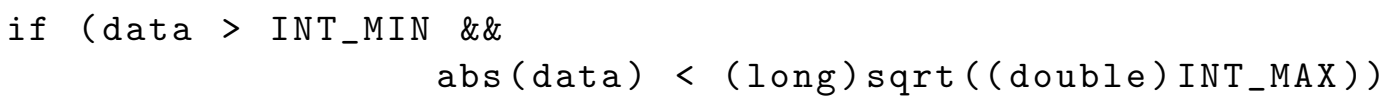

Variants of CWE190 cases comprise five operations: square (data*data), multiply $($ data $* 2)$, add $($ data +1$)$, prefix increment (++data), and postfix increment (data ++$)$. Only the cases with square operation had this problem. All others used simpler checks that did not have a problem with INT_MIN.

The case variants comprise five types: char, int64_t, int, short, and unsigned_int. The types char and short are fine, in usual architectures, because CHAR_MIN and SHORT_MIN do have positive int representations. Unsigned integers are all positive and thus not a problem. The problem with abs(min) only occurs for types int and int64_t. Type int64_t variants had additional problems, so we discuss them in the next section, Sec. 2.12. Type unsigned int variants have a different problem, so we discuss them in Sec. 2.13.

In 30 test cases, the "input method" is just the hardcoded value INT_MAX. The following example old good code is from CWE190_Integer_Overflow__int_max_square_01.c deprecated 83646:

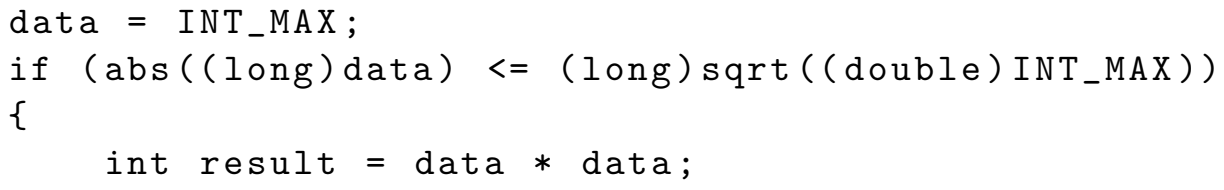

Eric Trapnell argued that because data can never be INT_MIN in these cases, the check need not be changed. Since these tests are for static analyzers, it may be informative to have as consistent code as possible, in case a known value of INT_MAX affects analysis. Accordingly, we changed those cases, too.

Using a hardcoded value to "fix" the good function may be a concern. This changes the functionality and is therefore not a fix as much as it is removing the bug. See Sec. 3.8 for more discussion about this.

For int type, we fixed $288 \mathrm{C}$ files (in 288 test cases) under CWE190 under s02 (48 files), s03 (192 files), and s04 (48 files). A total of 228 files were in C, and 60 files were in $\mathrm{C}++$. These were all named CWE190_Integer_Overflow_-int_*_square_*.c or .cpp.

Pascal Cuoq reported this problem on 25 July 2013 (comment N). André Maroneze reported this problem on 12 June 2017 (suggestion 3). This problem was Eric Trapnell's row 14. 
After Juliet 1.3 was released, Maroneze reported that many cases still had similar problems with range checks. We provide details in Sec. 3.5.

\subsection{Wrong Check for Value Out of Range - int64_t}

The old Juliet Version 1.2 code does not properly handle int64_t types, even with casts. The following example old good code is from CWE190_Integer_Overflow_-int64_t_ fscanf_square_01.c deprecated 82638:

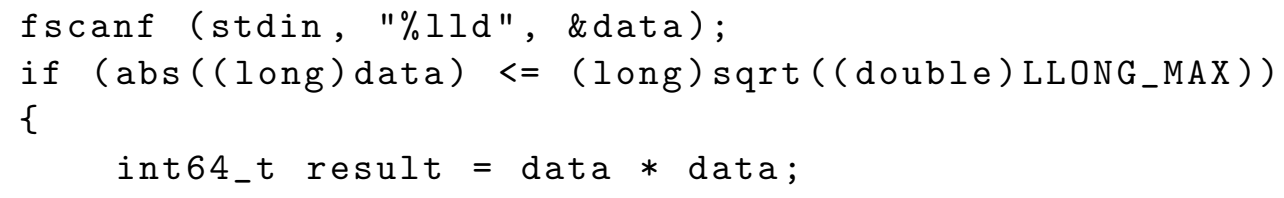

The functions abs() and sqrt() do not handle int64_t type values. The cast to double may distort values in other ways. The following new check is from 235405:

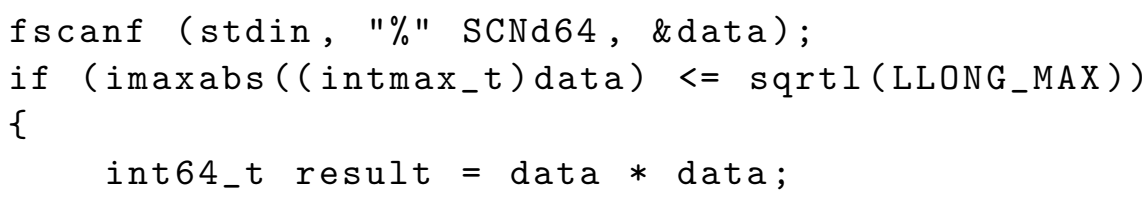

We also added an include of inttypes. $h$ to all the files of these cases.

We also noticed that the \%lld format specifier does not handle int64_t type values. We corrected it.

For int64_t type, we changed $178 \mathrm{C}$ and C++ files (in 144 test cases) under CWE190 s02. Of those, 114 files were $\mathrm{C}$, and 30 files were $\mathrm{C}++$. These files were all named CWE190_Integer_Overflow_-int64_t_*_square_*.c or .cpp.

The number of files fixed for int64_t type cases differs from the number fixed for the int type cases (Sec. 2.11). The int cases have input variants connect_socket, fgets, and listen_socket. The int64_t type cases do not have those input variants. Also, we changed int64_t files to fix a scanf () format specifier problem (Sec. 2.15).

Pascal Cuoq reported this problem on 25 July 2013 (comment P). This problem was Eric Trapnell's rows 14 and 16.

As we wrote this report, we realized that this fix does not solve the minimum integer problem, as explained above in Sec. 2.11. We note this as a problem remaining in Juliet 1.3 in Sec. 3.4.

After Juliet 1.3 was released, André Maroneze reported that many cases still had similar problems with range checks. We found 432 cases in Juliet 1.3 that we failed to fix. We provide details in Sec. 3.5.

As with the int cases, there are still a concern with some of these cases. One version of the good function is "fixed" by using a hardcoded value: data = LLONG_MAX. Sec. 3.8 discusses this in more detail. 


\subsection{Wrong Check for Value Out of Range - unsigned int}

This check is, to paraphrase Pascal Cuoq, a theoretical problem that would only happen on an architecture with a 64-bit int type, which is rare. The following old checking code is from CWE190_Integer_Overflow_-unsigned_int_fscanf_square_01.c deprecated 84366:

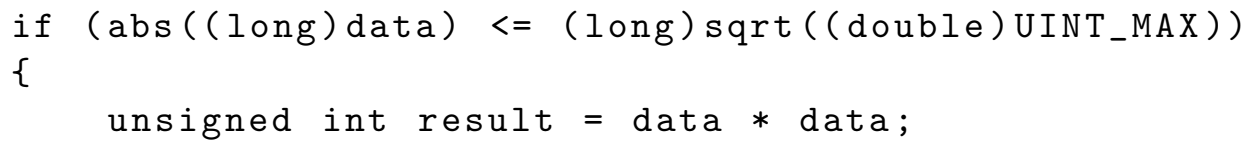

When UINT_MAX $\left(2^{64}-1\right)$ is converted to double, it is rounded up to $2^{64}$. The square root of $2^{64}$ is $2^{32}$. If data is $2^{32}$, it passes the check, and the multiplication overflows.

We followed Cuoq's recommendation and changed $<=$ to $<$. The following example new code is from 235789:

if (abs ( (long)data) < (long) sqrt ( (double)UINT_MAX))

We changed 144 files under CWE190 s05, all named __unsigned_int_*_square_.

Pascal Cuoq reported this problem on 25 July 2013 (comment O). This problem was Eric Trapnell's row 15.

\subsection{Wide Format Strings Mishandled}

Some test cases pass wide character format strings to snprintf(), which does not handle them. The following old code is from CWE122_Heap_Based_Buffer_Overflow_-_ CWE805_wchar_t_snprintf_01.c deprecated 72176:

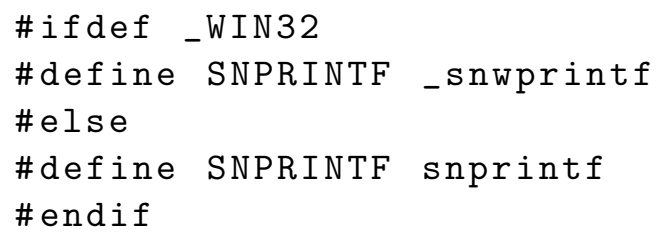

We replaced snprintf() with swprintf() in 672 files. There are 188 files under CWE121 (in s05, s06, s07, and s08), 208 files under CWE122 (in s04, s05, s09, and s10), 240 files under CWE134 (in s03, s04, s05, and s06), 18 files under CWE252, and 18 files under CWE253. All of the cases have _wchar_t_ in their names and have _snprintf just before the variation number.

Pascal Cuoq reported this problem on 26 June 2013 (comment K). This problem was Eric Trapnell's row 12.

As we wrote this report, we noticed that source itself is a wide string. It is handled with the wrong format specifier: $\%$ s instead of $\%$. This is a latent bug in Juliet 1.3, which we explain in Sec. 3.6. 


\subsection{Wrong $f \operatorname{scanf}()$ Format Specifier for int64_t and size_t}

The $\% 1 l d$ format specifier, used in $f \operatorname{scanf}()$, does not handle int64_t type variables. The following example old code is from CWE190_Integer_Overflow_-int64_t_fscanf_ square_01.c deprecated 82638 :

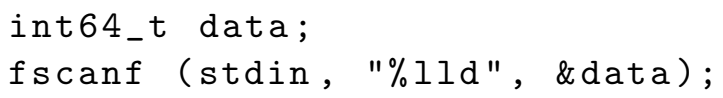

We changed the code to use SCNd64. The following new input code is from 235405:

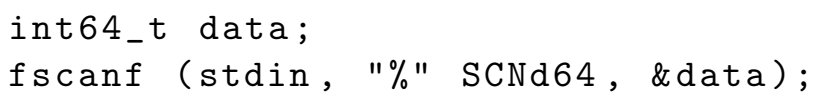

We also added an include of inttypes. $h$ in all the files of these cases. This occurs in 352 files in the following seven subdirectories: CWE190, subdirectories s01, s02, s06, and s07, and CWE191, subdirectories s01, s04, and s05. The files are named CWE19[01]_Integer \{Over, Under\}flow_-int64_t_fscanf_FUNC_*, where FUNC is add, sub, multiply, preinc, postinc, predec, or postdec.

Similar to that, the \%ud format specifier is not the standard way to handle size_t variables. The following example of old code is from CWE789_Uncontrolled_Mem_Alloc__new_ char_fscanf_14.cpp deprecated 117572:

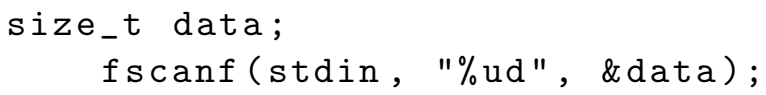

We replaced \%ud with \%zu. The following new code is from 243952 :

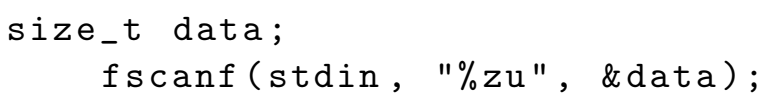

These are all under CWE789. The cases are named CWE789_Uncontrolled_Mem_Alloc $\left\{\mathrm{malloc}, \mathrm{new}_{-}\{\mathrm{char}\right.$, wchar_t\}_fscanf_*. $\{c, \mathrm{cpp}\}$. The _-malloc_cases are in $\mathrm{s} 01$. The _-_new_ files are in s02. There are 100 files in each of s01 and s02.

André Maroneze reported this problem on 12 June 2017 (part of suggestion 2). This problem was Eric Trapnell's row 17.

Similar to those two problems, utility functions in the support file io.c used the wrong format specifiers. The following is the old code:

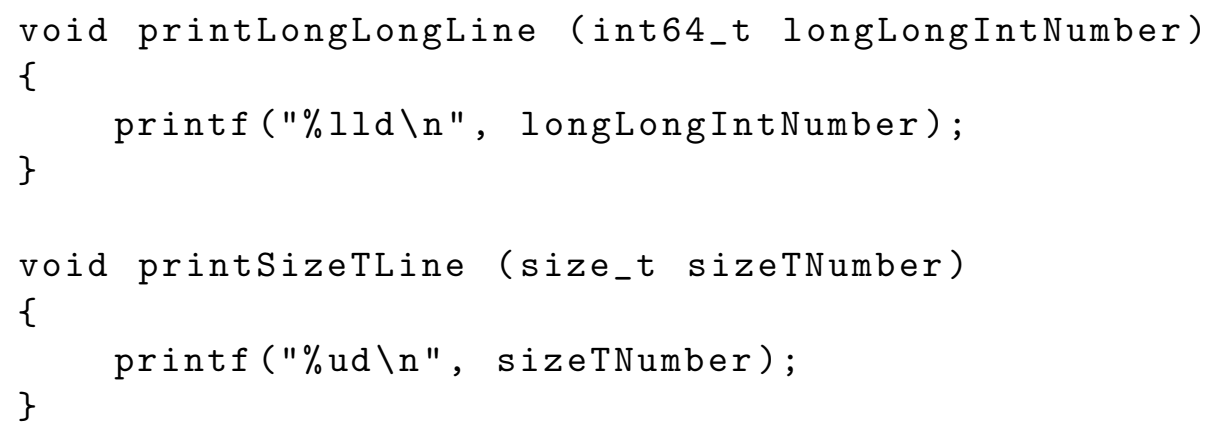


We changed this code to use the appropriate format specifiers:

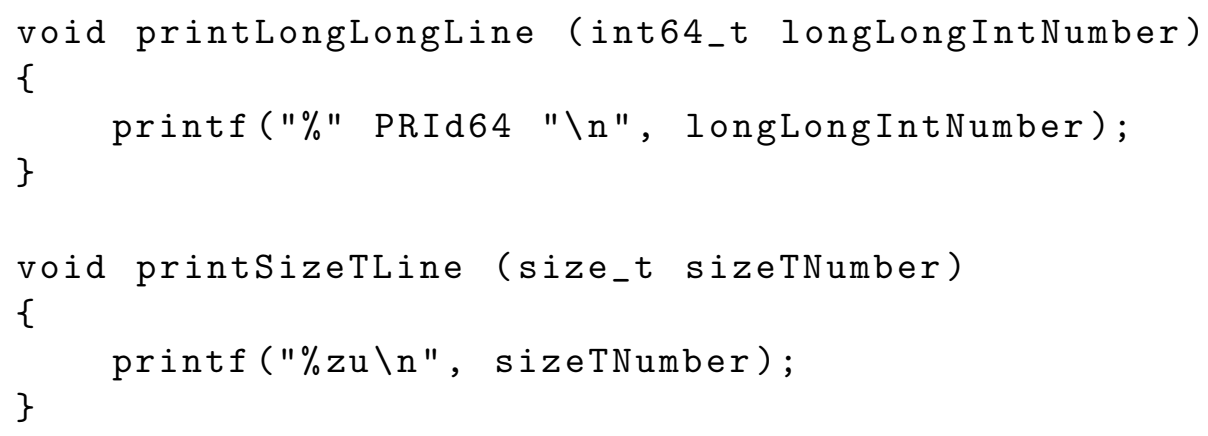

We also added an include of inttypes.h, to define PRId64, and wctype.h, to declare iswxdigit().

Pascal Cuoq reported this problem as part of comment Q.

\subsection{Improve Compile Files and Scripts}

As explained in [5] and [4], Juliet was designed so that the user could either compile all test cases in one big executable $(A l l)$ or each test case as its own, individual executable (Ind).

The Juliet 1.2 makefile command to make one big executable, $A l l$, for $\mathrm{C} / \mathrm{C}++$ on a Unix platform was impractical. The command passed more than 100000 files to gcc, which gcc could not handle.

As one step to fix that, we changed the makefile command to generate individual test case executables, Ind, (using -DINCLUDEMAIN), in addition to one executable for each CWE. (One executable per CWE was already available in Juliet 1.2.) We changed this command to create unlinked object files (.o files) for each source file (.c or .cpp files). Following that all of the object files in a directory could be linked to create an executable of all of the test cases in that directory or all of the object files could be linked to create a partial .o file.

The partial.o in each directory is used by the new makefile command to link the one big executable, All. We heavily edited the command to link each directory's partial.o. This compilation process is far quicker than the process in Version 1.2 and consumes fewer resources.

Hence in the top directory, there are now two make commands:

$\$$ make individuals

creates an executable file for each $\mathrm{C} / \mathrm{C}++$ test case.

$\$$ make Juliet1.3

or

$\$$ make

invokes make individuals, then creates a single executable with of all the test cases.

Similar options are supported for Windows in the .bat files, except that individual executables are not supported. 
For the Java code, the most significant improvement is that there are now commands available in the top directory for compiling test cases:

$\$$ ant compile

creates only bytecode files.

$\$$ ant jar

creates java archive files.

$\$$ ant war

creates web archive files.

We also made small changes to improve the manifest of jar files.

For both $\mathrm{C}$ and Java, we removed code that is not needed. Previously scripts in the top directory had code to ignore directories named svn. SVN is a source control system, like git or rcs. In SVN, a .svn subdirectory stores change metadata for each source code directory. Hence, .svn directories were scattered all over. Files in those directories should not be included in makefiles or other files. Since there are no .svn directories in Juliet 1.3, the code is unnecessary.

\section{Known Problems in Juliet 1.3}

We corrected many problems in the Juliet 1.2 test suite. This section details the many systematic problems remaining in Juliet 1.3 of which we know. We decided not to fix some because the effort to fix the code exceeded any benefit. Four problems were noticed only months after Juliet 1.3 was released. They are detailed in Sections 3.3, 3.4, 3.5, and 3.6.

\subsection{Memory Leaks}

The first problem is that thousands of cases have memory leaks. That is, memory is allocated, but never freed (until the program ends). The following example is from CWE758_ Undefined_Behavior__double_pointer_alloca_use_01.c 112082:

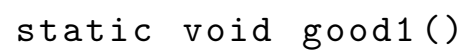


We decided not to fix this memory leak problem for several reasons. First, the memory leak does not lead to undefined behavior, and therefore should not stop analyzers from looking for other problems. Second, it should not cause any problem in practice. Even if all the cases are compiled and executed together, only about 2 megabytes of memory will be allocated.

Third and most importantly, it would take a huge amount of manual effort to write and test scripts to insert the proper free() commands at the right places. Worse, we foresee two mistakes possible with the scripts: the memory is freed too early or the wrong memory is freed. Either way, later code accesses freed memory. There is no simple way to check for these mistakes.

Aurélien Delaitre reported this problem on 15 September 2015. This problem was Eric Trapnell's row 19.

\subsection{No Evident Failure}

Although most cases have faults, i.e., corrupted internal states, many cases do not take user input or do not have externally apparent failures. The following bad code is from CWE416_Use_After_Free__malloc_free_char_01.c 240263:

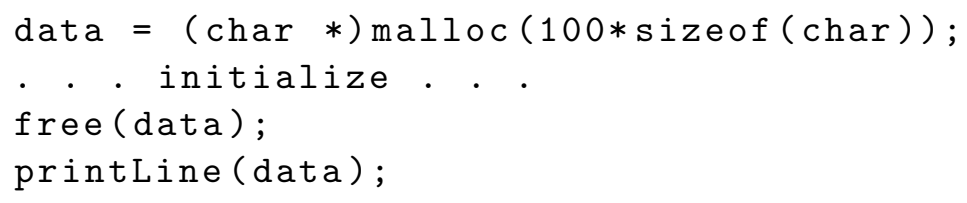

Although this code always uses memory after it is freed, there is usually no visible failure, e.g., a crash or corrupted result. Even some cases that have apparent failures do so for all inputs. That is, they crash given essentially any input.

Future versions of these test cases might be built so that all of them execute reasonably for some inputs and fail for other inputs. Even better would be versions that have exploitable security vulnerabilities.

David Musliner reported this problem on 28 February 2018.

\subsection{Memory Accessed After Its Lifetime}

All 80 cases under CWE843 access automatically allocated memory after its lifetime. The following example code is from CWE843_Type_Confusion__short_01.c 122807:

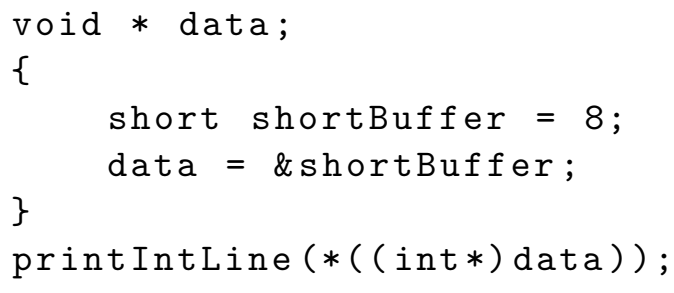

Memory for shortBuffer is automatically allocated (usually on the stack) by the time that execution enters the block. Although the address is saved, the lifetime of the memory ends 
when execution leaves the block. Thus, the argument to printIntLine() is an invalid dereference.

This can be fixed by declaring the variable earlier, as detailed in Sec. 2.6. This is how the fixed code might appear:

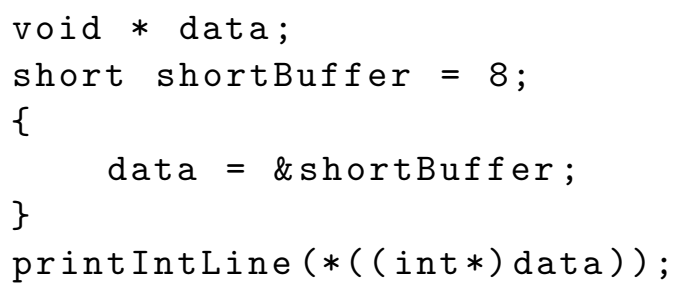

André Maroneze reported this problem on 12 April 2018.

\subsection{Check for Value Out of Range Still Wrong - int64_t}

As we wrote this report, we realized that the range check fix explained in Sec. 2.12 does not solve the minimum integer problem for int64_t. In brief, the absolute value of LLINT_MIN is not defined by the C11 standard [16]. The check should be

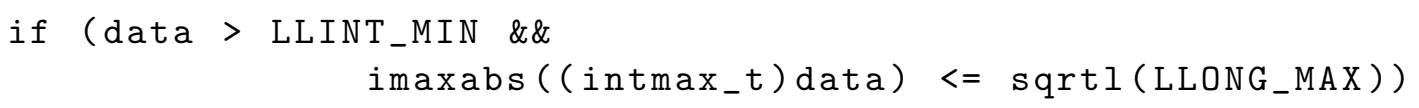

This affects 144 files under CWE190 s02. All the files with this problem are named CWE190*_int64_*square*.

\subsection{Additional Cases with Wrong Check for Value Out of Range}

Some of the good code under CWE190_Integer_Overflow intended to avoid overflow by checking that the value was in range. The following example of good code is from CWE190_ Integer_Overflow__unsigned_int_max_square_01.c 235831:

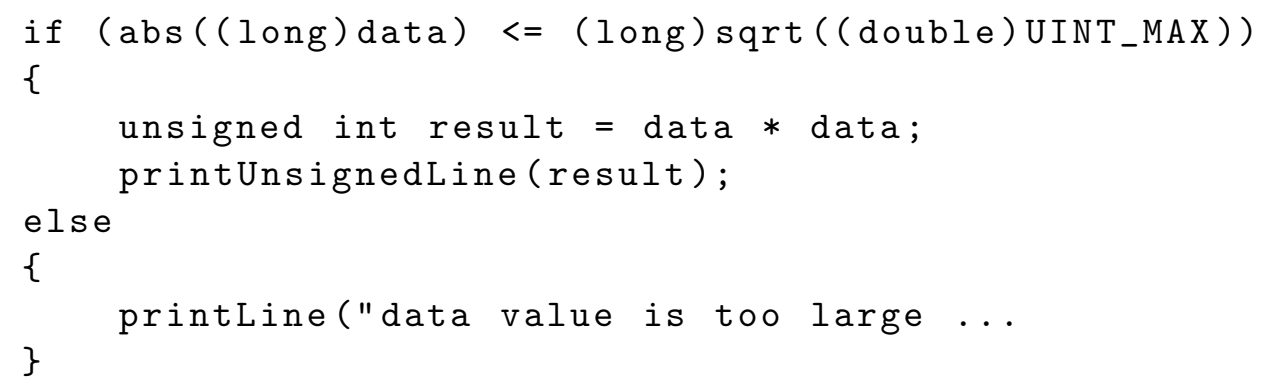

The problem is in abs ( (long)data). On some architectures, the largest unsigned integer will not fit in a long. Thus the cast does not behave as intended. In addition, abs () does not handle long values. The problem could be addressed by checking that the value fits and using an appropriate absolute value function, as explained in Sections 2.11 and 2.12. André Maroneze suggested the following code:

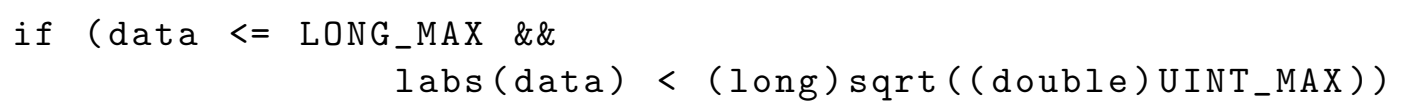


The files with this problem are all under CWE190, subdirectories s01, s04, and s05. They are named CWE190_Integer_Overflow__TYPE_SOURCE_square_VARIANT.c or .cpp, with three types, char, short, and unsigned_int, three sources, max, rand, and fscanf, and 48 flow variants, for a total of 432 files.

André Maroneze reported this problem on 12 April 2018.

\subsection{Wrong Format Specifier for Wide String}

As we wrote this report, we noticed the change explained in Sec. 2.14 does not fix all the printing problems. The following code is from CWE122_Heap_Based_Buffer_Overflow_-_ _ CWE805_wchar_t_snprintf_01.c 233407:

\#define SNPRINTF swprintf

wchar_t source [100];

SNPRINTF (data, 100, L"\%s", source);

The argument, source, is a wide string. The code has the wrong format specifier: \%s. To handle wide strings, the specifier should be $\% \mathrm{~s}$.

This problem needs to be fixed in 672 files under various directories: CWE121 (subdirectories s05, s06, s07, and s08), CWE122 (subdirectories s04, s05, s09, and s10), CWE134 (subdirectories s03, s04, s05, and s06), CWE252, and CWE253.

\subsection{Wrong Format Specifier to Print char as Hexadecimal}

In Juliet 1.3, utility code prints char type arguments with the wrong format specifier. The function printHexCharLine() is defined in the support utility file io.c as:

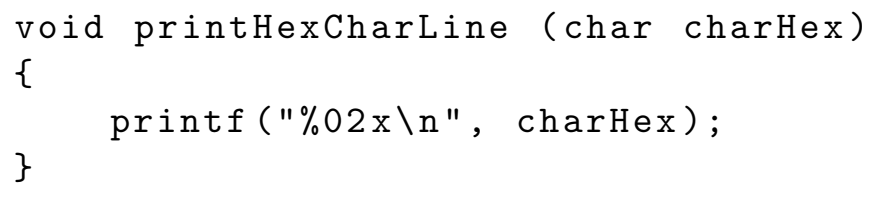

The format specifier $\% \mathrm{x}$ is for an unsigned int argument. In this case, charHex is promoted to int. If type char is signed, a common choice that the C11 standard [16] leaves to each compiler, a negative value is promoted to a negative integer by sign extension. That means the above typically prints 255 as ffffffff.

Pascal Cuoq suggested using casts to print properly: printf ("\%02x\n", (unsigned int)( unsigned char) charHex);

The cast (unsigned int) is needed because the C11 standard 7.21.6.1:9 "The fprintf function" states, "If any argument is not the correct type for the corresponding conversion specification, the behavior is undefined."

As an alternative, C11 7.21.6.1:7 provides a length modifier, hh, to handle this:

$$
\text { printf ( } \% 02 \mathrm{hhx} \backslash \mathrm{n} ", \quad \text { charHex); }
$$


Pascal Cuoq reported this problem as part of comment Q. André Maroneze reported this problem on 12 June 2017 (part of suggestion 2).

\subsection{Many Bugs Removed by Using Hardcoded Values}

The most widespread and challenging issue in Juliet 1.3 is that the good code in thousands of cases removes a problem by just using a hardcoded value. For instance, some cases under CWE190 read a value then increment it. The following example bad code is from CWE190_Integer_Overflow__int_fscanf_add_01.c 83262:

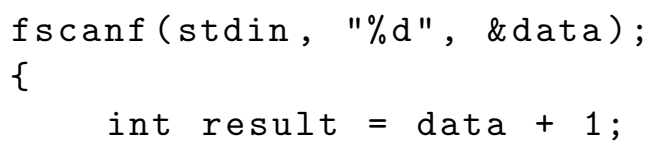

Note that there is no check for overflow. This particular case has two good functions, each with a different resolution. One adds a check:

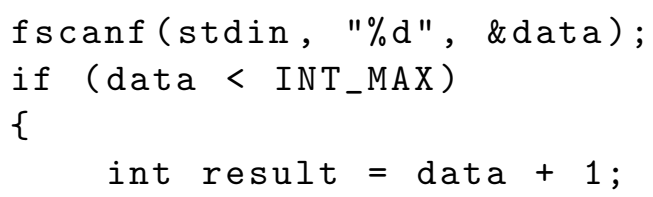

The other one merely sets the variable to a value that will not cause an overflow:

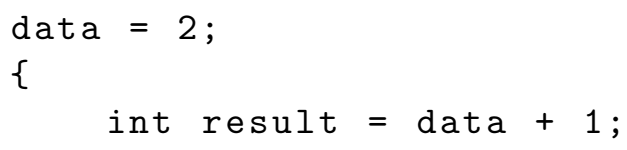

Hardcoded values to remove bugs are located throughout the Juliet test suite. For instance, cases under nineteen CWEs use

$$
\text { data }=\text { "foo"; }
$$

We identified a dozen different kinds of hardcoded constants, such as integers, passwords, and strings.

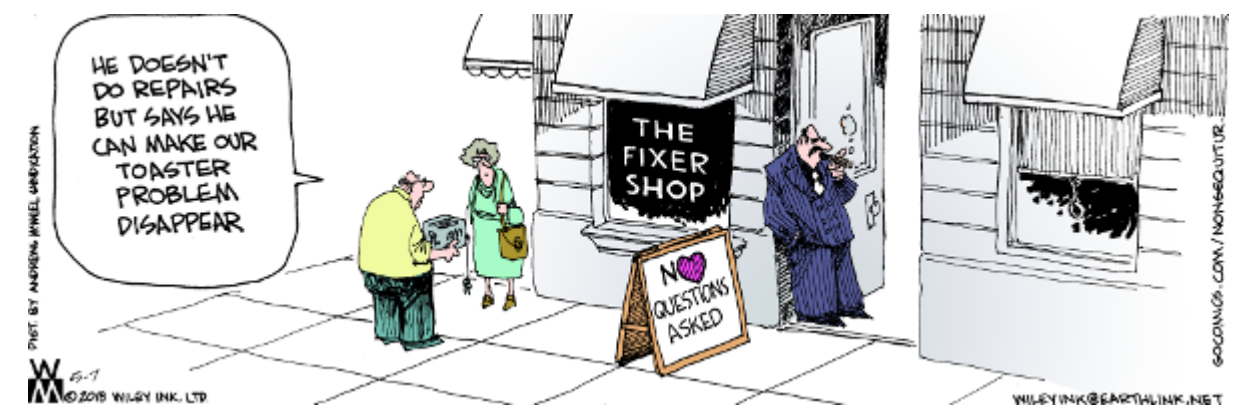

Fig. 1. A cartoon highlighting that making a bug disappear is not the same as fixing it. NON SEQUITUR () 2018 Wiley Ink, Inc.. Dist. By ANDREWS MCMEEL SYNDICATION. Reprinted with permission. All rights reserved. 
To test that a static analysis tool recognizes the basic difference between a vulnerability and no vulnerability, the use of hardcoded "inputs" is reasonable acceptable. But eliminating the problem using a hardcoded value changes the program behavior (drastically!). These "fixes" are very different from the patches one would find in real code.

Damien Cupif points out that these pseudo-fixes tend to invalidate discrimination calculations. That is, the behavior of the good version is significantly different than the bad version behavior, not just for buggy values.

Expanding the utility of the Juliet suite may require rethinking the tactic of eradicating problems with hardcoded values. Finding a good resolution would require extensive consideration and changing thousands of files in a dozen different ways.

\subsection{Uncaught Java Exceptions}

We know of at least two uncaught Java exceptions in Juliet 1.3. In the following example, the constructor OutputStreamWriter can throw an exception that is not caught, creating a potential resource leak. The following example code is from CWE400_Resource Exhaustion_-getParameter_Servlet_write_72b.java 138404:

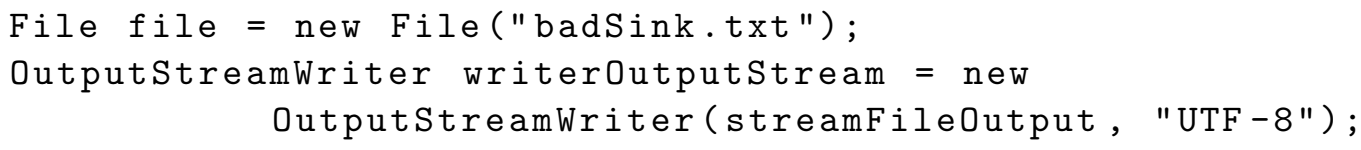

If the Java installation does not support UTF-8, the method exits, but the file remains open. This problem was Eric Trapnell's row 3.

Similarly, the constructor InputStreamReader can throw an exception. The resource is a URL connection in this example from CWE400_Resource_Exhaustion__URLConnection_ for_loop_14.java 139105:

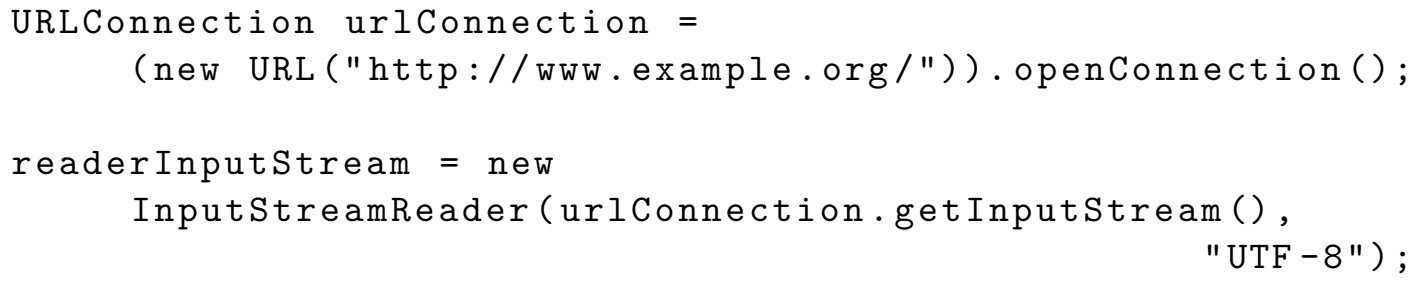

These are unlikely to cause problems in any execution of these test cases.

Aurélien Delaitre reported both of these problems in connection with SATE V [17]. This problem was Eric Trapnell's row 4.

\subsection{Dead Stores}

Forty cases have unintentional dead stores. That is, a value is stored in a variable, and the value is overwritten before it is used. There is no way to fix these and still keep the code similar to other variants. For example, here is bad code that does not use a variable. It is from CWE563_Unused_Variable__unused_uninit_variable_char_33.cpp 105689: 


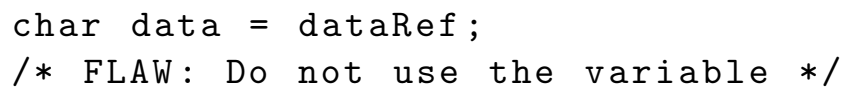

The good code initializes then prints data.

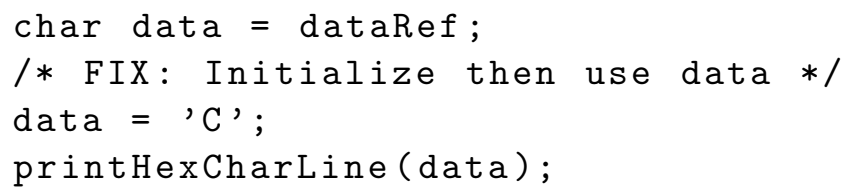

This occurs for two scenarios (unused_uninit_variable and unused_value), four types (int, char, wchar_t, and long), and four code variants (33, 72b, 73b, and 74b). The corresponding files are named CWE563_Unused_Variable__SCENARIO_TYPE_VARIANT.cpp.

We could not think of a way to fix the dead store so that they are still similar to the 01 variants and also have the same structure as the thousands of other 33 variants. One approach is just to remove these tests: there are many other test cases with unused variables, unused values, dead stores, etc. Another approach is to not declare the local variable and use the right hand side of the assignment instead. So the above good code would become:

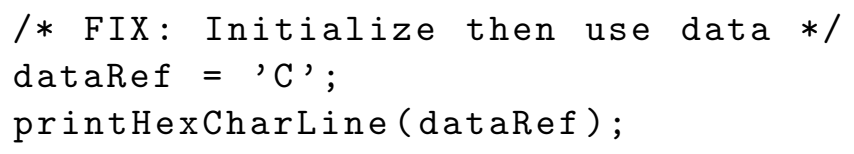

We decided to leave the dead stores and note them as extraneous weaknesses.

Aurélien Delaitre reported this problem on 6 August 2015. This problem was Eric Trapnell's row 18.

\subsection{Dead Code not in Metadata}

There are thousands of cases with dead code, but the metadata that accompanies test cases and test suites does not note it. The following example code is from CWE190_Integer Overflow_-int_File_postinc_02.java 249242:

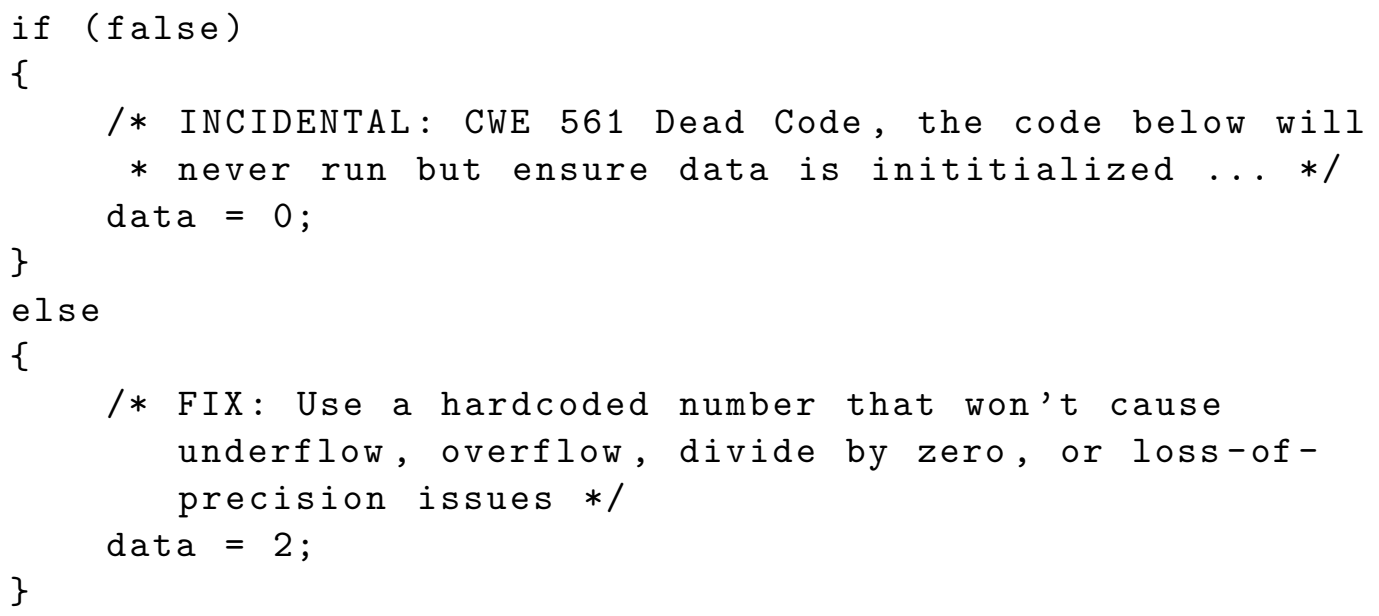

For consistency and completeness, such dead code should be noted in the metadata for automated checking. 


\subsection{Integer Overflow not in Metadata}

CWE-680 is a chain of two faults, an integer overflow (FRS/Overflow) that leads to a BOF/Write. All cases under CWE680 have metadata for BOF/Write at the right line. However, the FRS/Overflow is not in the metadata. The following example code is from CWE680_Integer_Overflow_to_Buffer_Overflow__malloc_rand_01.c 241054:

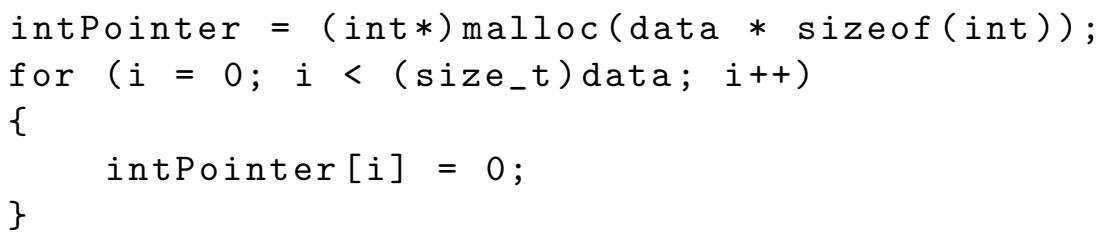

The computation for the amount of memory to allocate may overflow, causing a buffer that is too small to be allocated.

\subsection{Temporary Files Still Not Secure}

In Sec. 2.10, we explained how temporary files in Juliet 1.3 are more secure than those in Juliet 1.2. There we also explained the two remaining problems. We mention them again here to list all known problems in a single section.

First, we did not change any wchar_t cases because there is no widely-used equivalent of mkstemp() that handles wchar_t names.

Second, mkstemp () is not entirely secure. The file names are too predictable.

\subsection{Suggestions We Did Not Take}

In addition to comments and known problems, we received a number of suggestions on which we did not take action. This section records them.

\subsubsection{Add RAND16 and RAND8 Macros}

In 402 files of Juliet Version 1.3, data is assigned a random char value. In other files, data is assigned a random short value. The following example code is from CWE190_Integer Overflow__char_rand_postinc_01.c 235991:

$$
\text { data }=(\text { char }) \operatorname{RAND} 32() \text {; }
$$

The cast narrows the integer returned by RAND32() to a char, which changes some values. The C11 standard states that the exact nature of the changes is left to the implementation. In suggestion 1, André Maroneze said that users frequently request that such cases of implementation-dependent behavior be reported. To make it clear that there is no problem intended in these cases, he suggested adding new macros, for example:

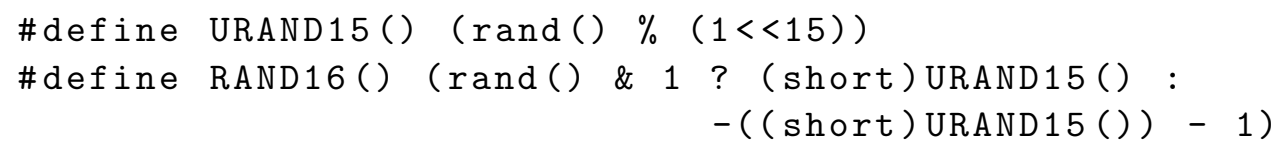




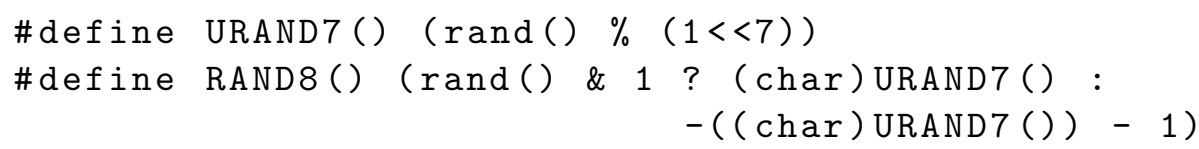

We improved existing macros for random numbers (Sec. 2.8). However, we did not add specific macros to produce random char or short values.

\subsubsection{Move Cases of NULL Check After Dereference}

As explained in Sec. 2.4, 18 cases intentionally check a pointer for NULL after the pointer is dereferenced. In suggestion 7, André Maroneze suggested moving these cases from CWE476_NULL_Pointer_Dereference to CWE571_Expression_Always_True. We decided that CWE-571 is not a sufficiently close match. In fact, there is no CWE for this problem.

This suggestion was Eric Trapnell's row 15.

\subsubsection{Move Cases of Incorrectly Calculating Multi-Byte String Length}

André Maroneze's suggestion 7 was to move cases of incorrectly calculating multi-byte string length to a new directory named for CWE-135 Incorrect Calculation of Multi-Byte String Length. The following example of Juliet 1.3 code is from CWE122_Heap_Based_ Buffer_Overflow__CWE135_01.c 232119:

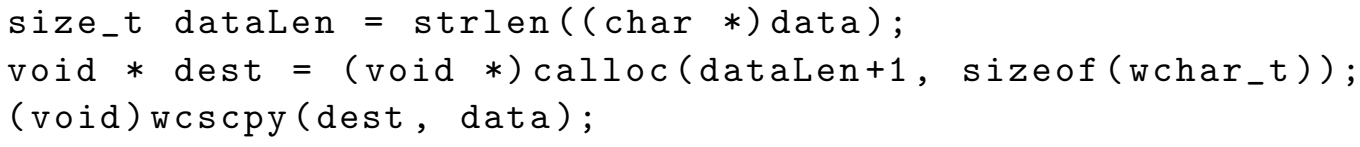

This affects 172 files in 96 cases, all with __CWE135_ in their names. They are all under CWE121 and CWE122.

The original catalyst for looking at them was that the cases did not have BOF [11]. This problem was fixed so that the cases have BOF (Sec. 2.2).

The misuse of strlen() on wide character strings is intentional. We decided not to create a new directory and move these cases there for several reasons. First, these are legitimate BOF cases, so there is reason to leave them where they are or to duplicate them. Second, if we did create a new directory, it should contain a thorough set of cases of incorrectly calculating, not just one example. For instance, perhaps the misuse should be in other contexts, like printing and reading, not just copying. Third, for consistency, we would rename all of the files to start with CWE135_* and change the names of the functions in the code. Finally, we plan to eventually replace the CWE classification with BF classification.

\section{Some Thoughts on the Future of Juliet and Test Suites}

In this section, we provide some thoughts on the future of the Juliet test suite and assurance tool testing in general. 
There is no plan to create a Juliet Version 1.4. On one hand, many known problems can be corrected with techniques used to create Version 1.3. On the other hand, the Center for Assured Software plans to generate future test suites on demand. That is, a custom set of tests will be generated for each user. Custom sets of tests reduce the incentive to code a tool to an unchanging test suite.

With oversight and direction by NIST, students at TELECOM Nancy, a computer engineering school of the Universite de Lorraine, Nancy, France implemented and then improved a test case generator [7]. They used the generator to create suites similar to Juliet for PHP and C\# [18].

What would be the ultimate test suite? As Cohen et. al. explained [17], a perfect collection has three aspects: it represents production software, we know where all the bugs are, and it has lots of different types of bugs in varied situations. Juliet incorporates the last two aspects. Software that is used in production is typically large and complex. Juliet cases are far smaller and less complex than production software. Most synthetic or generated collections will be similarly small and less complex.

An approach to achieving all three aspects is to inject bugs into production software. Automated tools can help by finding locations with desirable execution flow, program state, and data visibility. However, none of the published approaches appear to have a path to completely automated bug injection of many types of bugs.

\section{Acknowledgments}

We thank all those who reported problems in Juliet 1.2, especially Pascal Cuoq (Pascal.CUOQ@cea.fr), Takashi Matsuoka (takashi.matsuoka@redlizards.com), André Maroneze (Andre.OLIVEIRAMARONEZE@cea.fr), Bertrand Stivalet, Aurélien Delaitre, and Elisa Heymann (elisa@cs.wisc.edu). We also thank Eric Trapnell and Charles D. De Oliveira for their work on Juliet 1.3.

\section{References}

[1] Center for Assured Software (2012) Juliet Test Suite for C/C++ - Changelog. Accessed 19 March 2018. URL https://samate.nist.gov/SARD/view.php?tsID=86.

[2] Common weakness enumeration: A community-developed list of software weakness types. Accessed 4 January 2018. URL https://cwe.mitre.org/.

[3] Center for Assured Software (2012) Juliet Test Suite for Java - Changelog. Accessed 19 March 2018. URL https://samate.nist.gov/SARD/view.php?tsID=87.

[4] Center for Assured Software (2012) Juliet Test Suite v1.2 for Java User Guide. Accessed 19 March 2018. Also available with the Juliet Test Suite v1.3 for Java. URL https://samate.nist.gov/SARD/resources/Juliet_Test_Suite_v1.2_for_Java_ -_User_Guide.pdf. 
[5] Center for Assured Software (2012) Juliet Test Suite v1.2 for C/C++ User Guide. Accessed 19 March 2018. Also available with the Juliet Test Suite v1.3 for C/C++. URL https://samate.nist.gov/SARD/resources/Juliet_Test_Suite_v1.2_for_C_ Cpp_-_User_Guide.pdf.

[6] Software assurance reference dataset (SARD). Accessed 4 January 2018. URL https: //samate.nist.gov/SARD/.

[7] Black PE (2017) SARD: Thousands of reference programs for software assurance. Journal of Cyber Security and Information Systems - Tools \& Testing Techniques for Assured Software - DoD Software Assurance Community of Practice: Volume $25(3): 6-13$.

[8] Bojanova I, Black PE, Yesha Y (2017) Cryptography classes in bugs framework (BF): Encryption bugs (ENC), verification bugs (VRF), and key management bugs (KMN). 2017 IEEE 28th Annual Software Technology Conference (STC), pp 1-8. https://doi.org/10.1109/STC.2017.8234453. Gaithersburg, Maryland

[9] Boland T, Black PE (2012) Juliet $1.1 \mathrm{C} / \mathrm{C}++$ and Java test suite. IEEE Computer 45(10):88-90.

[10] Bojanova I, Black PE, Yesha Y, Wu Y (2016) The bugs framework (BF): A structured approach to express bugs. 2016 IEEE International Conference on Software Quality, Reliability, and Security (QRS), pp 175-182. https://doi.org/10.1109/QRS.2016.29. Vienna, Austria

[11] Buffer overflow (BOF) class. Accessed 20 March 2018. URL https://samate.nist.gov/ BF/Classes/BOF.html.

[12] Black PE, Ribeiro A (2016) SATE V Ockham sound analysis criteria. National Institute of Standards and Technology, NIST IR 8113. https://doi.org/10.6028/NIST.IR. 8113. Noted 23 June 2017.

[13] Injection (INJ) class. Accessed 22 March 2018. URL https://samate.nist.gov/BF/ Classes/INJ.html.

[14] dash - command interpreter (shell). Accessed 22 January 2018. URL http:// manpages.ubuntu.com/manpages/xenial/en/man1/sh.1.html.

[15] IEEE (2017) 1003.1-2017 Portable Operating System Interface (POSIX).

[16] (2011) ISO/IEC 9899:2011 programming languages - C, Committee Draft - April 12, 2011 N1570. The International Organization for Standardization and the International Electrotechnical Commission (ISO/IEC) Joint Technical Committee JTC 1, Information technology, Subcommittee SC 22, Programming languages, their environments and system software interfaces, Working Group WG 14 - C.

[17] Cohen TS, et al. (2017) Improving software assurance through static analysis tool expositions. Journal of Cyber Security and Information Systems - Tools \& Testing Techniques for Assured Software - DoD Software Assurance Community of Practice: Volume 2 5(3):14-22.

[18] Stivalet B, Fong E (2016) Large scale generation of complex and faulty PHP test cases. Proc. 2016 IEEE International Conference on Software Testing, Verification and Validation (ICST), pp 409-415. https://doi.org/10.1109/ICST.2016.43 